\title{
Mechanism underlying prolongevity induced by bifidobacteria in Caenorhabditis elegans
}

Tomomi Komura, Takanori Ikeda, Chikako Yasui, Shigeru Saeki \& Yoshikazu Nishikawa

\begin{tabular}{|c|l|}
\hline Citation & Biogerontology. 14(1); 73-87 \\
\hline Issue Date & $2013-02$ \\
\hline Type & Journal Article \\
\hline Textversion & author \\
\hline & $\begin{array}{l}\text { This is a post-peer-review, pre-copyedit version of an article published in } \\
\text { Biogerontology. The final authenticated version is available online at: } \\
\text { https://doi.org/10.1007/s10522-012-9411-6. }\end{array}$ \\
Rights & $\begin{array}{l}\text { See Springer Nature's AM terms of use: } \\
\text { https://www.springernature.com/gp/open-research/policies/accepted-manuscript-ter } \\
\text { ms }\end{array}$ \\
\hline DOI & \begin{tabular}{l}
$10.1007 / s 10522-012-9411-6$ \\
\hline
\end{tabular} \\
\hline
\end{tabular}

Self-Archiving by Author(s) Placed on: Osaka City University Repository 
1 Mechanism underlying prolongevity induced by bifidobacteria in

2 Caenorhabditis elegans

3

4 Tomomi Komura · Takanori Ikeda $\cdot$ Chikako Yasui $\cdot$ Shigeru Saeki $\cdot$ Yoshikazu

$5 \quad$ Nishikawa

6

7 T. Komura $\cdot$ T. Ikeda $\cdot$ C. Yasui $\cdot$ S. Saeki $\cdot$ Y. Nishikawa $(\bowtie)$

8 Department of Food and Human Health Sciences, Graduate School of Human Life Science,

9 Osaka City University, 3-3-138 Sugimoto, Sumiyoshi-ku, Osaka 558-8585, Japan

10 e-mail: nisikawa@life.osaka-cu.ac.jp

11 Tel. \& Fax: +81 6-6605-2910

12

13 
1 Abstract Lactobacilli and bifidobacteria are probiotic bacteria that modify host defense

2 systems and have the ability to extend the lifespan of the nematode Caenorhabditis elegans.

3 Here, we attempted to elucidate the mechanism by which bifidobacteria prolong the lifespan

4 of $C$. elegans. When the nematode was fed Bifidobacterium infantis (BI) mixed at various

5 ratios with the standard food bacterium Escherichia coli strain OP50 (OP), the mean lifespan

6 of worms was extended in a dose-dependent manner. Worms fed BI displayed higher

7 locomotion and produced more offspring than control worms. The growth curves of

8 nematodes were similar regardless of the amount of BI mixed with OP, suggesting that BI did

9 not induce prolongevity effects through caloric restriction. Notably, feeding worms the cell

10 wall fraction of BI alone was sufficient to promote prolongevity. The accumulation of protein

11 carbonyls and lipofuscin, a biochemical marker of aging, was also lower in worms fed BI;

12 however, the worms displayed similar susceptibility to heat, hydrogen peroxide, and paraquat,

13 an inducer of free radicals, as the control worms. As a result of BI feeding, loss-of-function

14 mutants of daf-16, jnk-1, aak-2, tol-1, and tir-1 exhibited a longer lifespan than OP-fed

15 control worms, but BI failed to extend the lifespan of pmk-1, skn-1, and $v h p-1$ mutants. As

16 skn-1 induces phase 2 detoxification enzymes, our findings suggest that cell wall components

17 of bifidobacteria increase the average lifespan of $C$. elegans via activation of $s k n-1$, regulated

18 by the p38 MAPK pathway, but not by general activation of the host defense system via

19 DAF-16.

21 Keywords Longevity $\cdot$ Nematodes $\cdot$ Probiotics $\cdot$ Aging $\cdot$ Innate immunity 
3 Age at infection is one of the most important determinants of disease morbidity and mortality

4 (Miller and Gay, 1997; Pop-Vicas and Gravenstein, 2011; Sligl and Majumdar, 2011).

5 Senescence of the immune system, which is a result of aging-related functional and metabolic

6 alterations in cells and tissues, leads to increased infections, malignancy, and autoimmunity

7 (Grubeck-Loebenstein, 1997; Moulias et al., 1985; Pawelec and Larbi, 2008). Elderly also experience increased mortality from viral and bacterial infections (Bradley and Kauffman, 1990). Slowing the senescence of immune function and decreasing mortality from infectious diseases may be possible through nutritional control; however, the difficulty of establishing a suitable animal model has limited studies on this topic. Although an increase of biomarkers related to immunological function has been observed in elderly with improved nutritional conditions (Bogden and Louria, 2004), few reports have shown beneficial influences of nutrition on immunity and the outcomes of experimental infection (Effros et al., 1991; Fulop et al., 2007; Hayek et al., 1997).

Probiotic bacteria are living microorganisms that exert beneficial effects on human health when ingested in sufficient numbers (Naidu et al., 1999). The concept of probiotic bacteria was first proposed in 1907 by Metchnikoff, who hypothesized that lactobacilli are important for promoting human health and longevity (Metchnikoff, 1907). Since this novel proposal over a century ago, lactic acid bacteria (LAB), including bifidobacteria, have been the most commonly used probiotic microorganisms. LAB have numerous physiological influences on their hosts, including antimicrobial, nutritional, anti-tumor, and immunomodulatory effects, microbial interference, and reduction of serum cholesterol and lipids. To date, however, few studies have examined the influence of LAB on longevity (Ottaviani et al., 2011). 
1 been extensively used as an experimental system for biological studies because of its

2 morphological simplicity, transparent body, ease of cultivation, and suitability for genetic

3 analysis (Riddle et al., 1997). In addition, the short and reproducible lifespan of C. elegans is

4 particularly advantageous for aging studies (Finch and Ruvkun, 2001). We previously

5 demonstrated that feeding nematodes LAB prolongs lifespan and enhances host defenses

6 (Ikeda et al., 2007; Komura et al., 2012). Further, we showed that Legionella pneumophila is

7 only pathogenic in older nematodes and that infection by this bacterium can be prevented by

8 feeding worms Bifidobacterium infantis (BI) before the infectious challenge (Komura et al.,

9 2010). However, neither the contributing bacterial factors nor the underlying mechanisms of

10 these beneficial effects have been clarified.

11 Caloric restriction is well known to extend the lifespan of a wide range of organisms from

12 yeasts to mammals (Ingram et al., 2006); however, it remains controversial whether caloric restriction increases the lifespan of primates (Austad, 2012). Here, we assessed whether bifidobacteria cause dietary or caloric restrictions in C. elegans based on the evaluation of worm biomarkers, including body and brood sizes (Pincus and Slack, 2010). While radical oxygen species (ROS) are recognized as key factors causing senescence because of their oxidative properties (Cannizzo et al., 2011), ROS are also considered to contribute to anti-senescence through the hormesis effect (Ristow and Schmeisser, 2011). Hence, we performed assays for muscle function, lipofuscin accumulation (gut autofluorescence), protein carbonyl content, stress resistance, and mitochondrial number to determine how bifidobacteria

21 induce the numerous beneficial effects on physiological function and longevity. In addition, 22 we evaluated whether the cell walls and protoplasts of BI contribute to the prolongevity of $C$. elegans. Finally, loss-of-function $C$. elegans mutants were fed BI to identify the genes involved in the probiotic effects in C. elegans. 


\section{Materials and methods}

4 Nematode

6 C. elegans Bristol strain N2 and its derivative mutant strains were kindly provided by the

7 Caenorhabditis Genetics Center, University of Minnesota. The mutations used in this study were IG10 tol-1(nr2033), RB1085 tir-1(ok1052), KU25 pmk-1(km25), EU1 skn-1(zu67), JT366 vhp-1(sa366), VC8 jnk-1(gk7), CF1038 daf-16(mu86), CB1370 daf-2(e1370), and RB754 aak-2(ok524). Nematodes were maintained and propagated on nematode growth 11 medium (NGM) according to standard techniques (Stiernagle, 1999). E. coli OP50 (OP) was used as the standard feed for nematode cultivation and was grown using tryptone soya agar

13 (Nissui Pharmaceutical, Tokyo, Japan). Cultured bacteria (100 mg wet weight) were 14 suspended in $0.5 \mathrm{ml} \mathrm{M9}$ buffer, and $50 \mu \mathrm{l}$ of the resulting bacterial suspension was then spread on peptone-free modified NGM (mNGM) in 5.0-cm diameter plates to feed worms.

Bacterial strains

The LAB B. infantis ATCC15697 (BI) used as a test food source for C. elegans and was cultured using GAM broth (Nissui) and TOS propionate agar (Yakult Pharmaceutical Industry, 21 Tokyo, Japan).

25 Eggs were recovered from adult $C$. elegans worms after exposure to a sodium 
1 hypochlorite/sodium hydroxide solution, as previously described (Sulston and Hodgkin, 2 1988). The egg suspension was incubated overnight at $25^{\circ} \mathrm{C}$ to allow hatching, and the 3 resulting suspension of L1 stage worms was centrifuged at $156 \times \mathrm{g}$ for $1 \mathrm{~min}$. After removing 4 the supernatant by aspiration, the remaining larvae were transferred onto fresh mNGM plates 5 covered with $\mathrm{OP}$ and then incubated at $25^{\circ} \mathrm{C}$. To synchronize pubescence, worms were 6 allowed to feed on OP for two days until maturation, as it is well known that the reproductive 7 system regulates aging in C. elegans (Hsin and Kenyon, 1999). Nematocidal assays were performed by adding 35 three-day-old adult worms to each mNGM plate covered with lawns of $\mathrm{BI}$. The plates were incubated at $25^{\circ} \mathrm{C}$, and the numbers of live and dead worms were scored every $24 \mathrm{~h}$. At $25^{\circ} \mathrm{C}$, worms produce progeny that develop into adults in 3 days and it is therefore difficult to identify the original worms. To avoid over-estimating the number of living worms, the original worms were transferred daily to fresh mNGM plates for 4 days until completing their egg-laying phase at 7 days of age. The worms were then transferred to fresh mNGM plates every second day. A worm was considered dead when it failed to respond to a gentle touch with a worm picker. Worms that died as a result of adhering to the wall of the plate were not included in the analysis. Nematocidal assays are generally performed using NGM agar plates containing peptone, which allows the overlayed bacteria to proliferate. However, the composition of NGM has been reported to influence the virulence of bacteria, and the in-situ production of metabolites by bacteria growing on the medium could also be fatal to nematodes (Anyanful et al., 2005). Thus, to exclude the possibility of 21 bacterial-induced nematocidal effects from nutrients in the medium, the nematocidal assays were performed on mNGM plates lacking peptone. Each assay was carried out in duplicate and repeated twice unless otherwise stated.

$$
\text { MLS }=\frac{1}{N} \sum_{j} \frac{x_{j}+x_{j+1}}{2} d_{j}
$$


1 where $d j$ is the number of worms that died in the age interval $\left(\mathrm{x}_{\mathrm{j}}, \mathrm{x}_{\mathrm{j}}+1\right)$, and $N$ is the total

2 number of worms. The standard error of the estimated mean lifespan was calculated using the

3 equation:

$4 \quad \mathrm{SE}=\sqrt{\frac{1}{N(N-1)} \sum_{j}\left(\frac{x_{j}+x_{j+1}}{2}-\mathrm{MLS}\right)^{2} d_{j} .}$

5 Maximum lifespan was calculated as the mean lifespan of the longest-living $15 \%$ of worms in 6 each group.

7

$8 \quad$ Locomotory scoring of ageing nematodes

10 The motility of worms at different ages was examined using a scoring method described in 11 previous reports (Gruber et al., 2011; Hosono et al., 1980). Briefly, worms were classified as 12 class "A" worms when they showed spontaneous movement or vigorous locomotion in responding to prodding; class "B" worms were those that did not move unless prodded or appeared to have uncoordinated movement; and class "C" worms were those that moved only their head and/or tail in response to prodding. Dead worms were classified as class "D". A minimum of 70 worms fed OP or BI were scored.

Lipofuscin

The autofluorescence of intestinal lipofuscin was analyzed as an indices of senescence from days 10 to 18 of adulthood. Briefly, randomly selected worms were washed five times in M9 buffer and then placed onto fresh mNGM plates coated with $50 \mathrm{mM}$ sodium azide to induce anesthesia. Lipofuscin autofluorescence was measured using a M165 FC fluorescence stereomicroscope (Leica Microsystems, Tokyo, Japan) equipped with a DsRED filter set 
1 (excitation, 510-560 nm; emission, 590-650 nm) and a Leica DFC425 C digital microscope

2 camera. The captured data was analyzed using Leica Application Suite imaging software 3 (Version 3.7.0).

Protein carbonyl formation

As biomarkers of oxidative stress, protein carbonyl groups were measured according to the method of Yang et al. (2007) with minor modifications. For each test group, 100 seven-day-old adult worms fed OP or BI for 4 days were picked, washed 3 times in M9 buffer, resuspended in $3 \mu \mathrm{l}$ M9 buffer, and stored at $-80^{\circ} \mathrm{C}$ until use. To lyse cells, $3 \mu 1$ lysis buffer (50 mM Tris- $\mathrm{HCl}$ buffer [pH 7.5], $150 \mathrm{mM} \mathrm{NaCl}, 0.1 \%$ SDS, $1 \%$ Triton X-100, $1 \mathrm{mM}$ PMSF, and $2 \%$ 2-mercaptoethanol) and $4 \mu \mathrm{l}$ of $15 \%$ SDS were added to the samples, which were then subjected to repeated freeze-thawing to release proteins. After centrifugation at $15,490 \times$ $\mathrm{g}$ for $3 \mathrm{~min}$, protein content was quantitatively measured using Bradford Ultra reagent (Expedeon Protein Solutions, Cambridge, UK). Detection of carbonyl groups was performed with the OxyBlot ${ }^{\mathrm{TM}}$ Protein Oxidation Detection Kit (Millipore, Billerica, USA). An equal volume of DNP solution (Oxyblot) or derivatization-control solution was added to samples, which were incubated at room temperature for 15 min. A volume of neutralization buffer (Oxyblot) equal to that of the DNP solution was then added to each sample. Samples containing one $\mu \mathrm{g}$ of total protein were then separated in $7.5 \%$ or $12 \%$ SDS-PAGE gels, transferred to PVDF membrane (GE Healthcare, Buckinghamshire, UK), and blocked with 1\% BSA for $1 \mathrm{~h}$. The membrane was incubated with rabbit anti-DNP antibody (Oxyblot) (1:150) for $1 \mathrm{~h}$, followed by goat anti-rabbit IgG (Oxyblot) (1:300) at room temperature for 1 h. Membranes were incubated with the ECL Plus detection reagent (GE Healthcare) and then 
1 Analysis Software. Band densities were analyzed using ImageJ software (developed by the

2 National Institute of Health). Membranes were incubated with $15 \%$ hydrogen peroxide for 30

3 min at room temperature and treated with anti-actin Clone $\mathrm{C} 4$ antibody (Millipore) to derive a

4 density value for actin for each lane unless otherwise stated. The measured Oxyblot values

5 were normalized to the density values determined for actin. Each assay was performed at least

6 twice for each sample.

7

8

9 determined. Each assay was performed with five plates and repeated twice.

Three-day-old adult worms were placed on mNGM plates covered with lawns of BI. The microscope (Shimadzu, Kyoto, Japan) and analyzed using ImageJ software. In this system, the area of a worm's projection was estimated automatically and used as an index of body size.

\section{Brood size}

Eggs isolated with a sodium hypochlorite/sodium hydroxide solution were allowed to develop to 3 days of age on $\mathrm{mNGM}$ plates coated with $\mathrm{OP}$ at $25^{\circ} \mathrm{C}$. Three hermaphrodites were selected and transferred to an mNGM plate covered with a lawn of BI. The parental animals The resulting progeny were left to develop for 3 days and the progeny number was then plates were incubated at $25^{\circ} \mathrm{C}$, and the body size of live worms were measured every $24 \mathrm{~h}$ until reaching 7 days of age. Images of adult nematodes were taken with a VCT-VBIT digital were transferred every $24 \mathrm{~h}$ to fresh mNGM plates until the end of the reproductive period. 
Stress resistance assays

2

3 Worms were grown from 3 to 7 days of age on mNGM plates with OP or BI and then

4 subjected to oxidative stress and heat shock assays. To conduct an oxidative stress assay,

5 worms were transferred onto mNGM containing 1.0 or $2.5 \mathrm{mM}$ paraquat or to $\mathrm{M} 9$ buffer plus

$60.1 \%$ cholesterol $\left(5 \mathrm{mg} \mathrm{ml}^{-1}\right.$ in ethanol) containing 0.8 or $2.0 \mathrm{mM}$ hydrogen peroxide,

7 incubated at $25^{\circ} \mathrm{C}$, and viability was then scored. To assess thermal tolerance, 7-day-old

8 worms were placed on M9 buffer or onto $\mathrm{mNGM}$ at $35^{\circ} \mathrm{C}$ and then scored for viability. The

9 survival of worms was determined by touch-provoked movement. Worms were scored as dead

10 when they failed to respond to repeated touching with a worm picker. The assays were

11 performed at least twice.

12

13 Quantification of mitochondria

14

Measurement of mitochondrial copy number was performed as described by Reinke et al. 16 (2010) with partial modification. Briefly, 3-day-old worms were placed in a 400- $\mu 1$ of M9 17 buffer containing $200 \mu \mathrm{g}$ 2'-deoxy-5-fluorouridine (Tokyo Chemical Industry, Tokyo, Japan), which was used to block the embryonic development of progeny and avoid contamination from offspring. The suspension was then spotted on mNGM in 9.0-cm diameter plates.

Worms were fed OP or BI for four days, recovered from the plates, and then washed five 21 times with M9 buffer. The worms were placed in lysis buffer $(0.2 \mathrm{M} \mathrm{NaCl}, 0.1 \mathrm{M}$ Tris- $\mathrm{HCl}$ [pH 8.3], $50 \mathrm{mM}$ EDTA, $0.5 \% \mathrm{SDS}$, and $100 \mu \mathrm{g} \mathrm{ml} \mathrm{m}^{-1}$ proteinase $\mathrm{K}$ ) and total DNA was then extracted using phenol/chloroform/isoamyl alcohol (25:24:1) and precipitated with ethanol.

24 Quantification of mitochondrial genes was performed using an ABI PRISM ${ }^{\circledR} 7000$ Sequence 
1 PCR Kit (Qiagen, Hilden, Germany) for $25-\mu 1$ reaction volumes containing $300 \mathrm{nM}$ of each

2 primer and $50 \mathrm{ng}$ of DNA template. Reactions were initiated at $95^{\circ} \mathrm{C}$ for $15 \mathrm{~min}$, followed by 340 cycles of $94^{\circ} \mathrm{C}$ for $15 \mathrm{~s}, 60^{\circ} \mathrm{C}$ for $30 \mathrm{~s}$, and $72^{\circ} \mathrm{C}$ for $30 \mathrm{~s}$. For quantifying mtDNA, the

$4 \quad c t b-1$ gene, which encodes cytochrome b of complex III, was targeted, while the atp-2 gene, 5 which encodes the b-subunit of the ATP synthetase complex, was selected for the nDNA 6 control. The primer sequences were as follows: $c t b-1$ forward, 5'-TGAAGCTGACCCTATAATGAGGC-3' and $c t b-1$ reverse, 5'-CCCCTAAGACTTTATTTGGAATAGCAC-3'; and atp-2 forward,5'-GCAACGTTCAGAAATGCGCT-3' and atp-2 reverse 5'-TGTtTGAGCTGAGGCGGACT-3'. Standard curves for mtDNA and nDNA were 11 generated using 10-fold serial dilutions of DNA plasmids containing cloned $c t b-1$ or atp-2 12 genes.

Cell wall fractionation of bifidobacteria

Cell walls were prepared as described by Tejada-Simon and Pestka (1999) with partial modification. Briefly, BI was anaerobically grown in GAM broth at $37^{\circ} \mathrm{C}$ for $48 \mathrm{~h}$. Cells were harvested by centrifugation at $14,000 \times \mathrm{g}$ for $10 \mathrm{~min}$ at $4^{\circ} \mathrm{C}$, washed once with sterile distilled water, and then centrifuged 3 times at $14,000 \times \mathrm{g}$ for $10 \mathrm{~min}$. The cells were heated at $100^{\circ} \mathrm{C}$ for $50 \mathrm{~min}$ for use as heat-killed bacteria. For preparation of the cell wall fraction, bacteria were disrupted by sonication for $30 \mathrm{~min}$ on ice using an UH-50 Ultrasonic Homogenizer (SMT, Tokyo, Japan). After heating the suspension at $60^{\circ} \mathrm{C}$ for $15 \mathrm{~min}$ to inactivate autolytic enzymes, the suspension was centrifuged at $800 \times \mathrm{g}$ for $30 \mathrm{~min}$ at $4^{\circ} \mathrm{C}$, and the pellet (unbroken cells) was removed. Cell walls were sedimented from the supernatant by centrifugation at $40,000 \times \mathrm{g}$ for $30 \mathrm{~min}$ at $4^{\circ} \mathrm{C}$ using an Optima ${ }^{\mathrm{TM}} \mathrm{MAX}-\mathrm{E}$ Ultracentrifuge 
1 (Beckman, Brea, CA, USA). The pellet was further treated with PRONASE ${ }^{\circledR}$ Protease $(0.78$ 2 units $\mathrm{mg}^{-1}$ of crude cell wall) (Merck, Darmstadt, Germany), ribonuclease A (250 $\left.\mu \mathrm{g} \mathrm{ml}^{-1}\right)$ 3 (MP Biomedicals, Illkirch, France), and DNAse I (250 $\left.\mathrm{g} \mathrm{ml}^{-1}\right)$ (Wako, Osaka, Japan) in 0.1

$4 \mathrm{M}$ Tris- $\mathrm{HCl}$ buffer $(\mathrm{pH} 7.4)$ at $37^{\circ} \mathrm{C}$ overnight to eliminate contaminating cytoplasmic 5 material. The treated cell wall fraction was centrifuged at $800 \times \mathrm{g}$ for $30 \mathrm{~min}$ at $4{ }^{\circ} \mathrm{C}$ followed 6 by centrifugation at $40,000 \times \mathrm{g}$ for $30 \mathrm{~min}$, weighed, heated to $90^{\circ} \mathrm{C}$ for $15 \mathrm{~min}$, and then resuspended at a concentration of $200 \mathrm{mg} \mathrm{ml}^{-1}$ in M9 buffer. The cell wall solutions were filter sterilized through a $0.45-\mu \mathrm{m}$ filter and kept at $-45^{\circ} \mathrm{C}$ until used as feed for C. elegans.

Bifidobacteria protoplast formation

Protoplast formation was performed as described elsewhere with minor modifications 13 (Lee-Wickner and Chassy, 1984). Briefly, protoplast formation buffer (20 mM HEPES (2-[4-(2-Hydroxyethyl)-1-piperazinyl] ethanesulfonic acid), $1 \mathrm{mM} \mathrm{MgCl} 2,0.5 \%$ gelatin, and $500 \mathrm{mM}$ lactose as a stabilizer; $\mathrm{pH}$ 7.0) was prepared and filter sterilized through a $0.45-\mu \mathrm{m}$ filter. BI cells anaerobically grown on TOS propionate agar at $37^{\circ} \mathrm{C}$ for $48 \mathrm{~h}$ were harvested using a platinum loop, washed thrice in M9 buffer, and suspended at $10 \mathrm{mg} / \mathrm{ml}$ in the protoplast formation buffer supplemented with $25 \mu \mathrm{g} \mathrm{ml} \mathrm{m}^{-1} \mathrm{~N}$-acetylmuramidase SG (Seikagaku Biobusiness, Tokyo, Japan) and $1 \mathrm{mg} \mathrm{ml}^{-1}$ lysozyme (MP Biomedicals). The resulting mixture was incubated at $37^{\circ} \mathrm{C}$ for $3 \mathrm{~h}$, and the cells were then washed 3 times with protoplast formation buffer. Protoplast formation was confirmed by the Gram negative-like appearance of cells following Gram staining, the KOH method (Gregersen, 1978), and the quantification of peptidoglycan using the SLP Reagent Set (Wako).

Statistical analysis 
2 Nematode survival was calculated by the Kaplan-Meier method, and survival differences 3 were tested for significance using the log-rank test. Differences in protein carbonyl levels and

4 brood size were determined using the Student's $t$-test. Differences in lipofuscin levels were 5 analyzed using the Mann-Whitney's U test. Differences in body sizes were analyzed using the 6 Kruskal-Wallis test. The level of significance for survival analysis was set at $\mathrm{P}<0.01$, as 7 indicated.

\section{Results}

Prolongevity effects of bifidobacteria

To ascertain whether the prolongevity effect of bifidobacteria on C. elegans is dose dependent, young adult worms fed OP for 2 days after hatching were transferred to plates containing BI mixed with OP at various ratios. Under these conditions, the lifespan of nematodes was significantly extended with increasing BI concentration (Table 1, Fig. 1).

In C. elegans, muscle function and accumulation of lipofuscin, as determined by gut autofluorescence, correlate with age, but vary between aged-matched individuals (Pincus and Slack, 2010). Here, locomotory ability was assayed as an indicator of muscle function, as

21 locomotory class is predictive for the remaining lifespan of C. elegans worms after 8 days of age (Hosono et al., 1980). The ratio of worms displaying coordinated sinusoidal locomotion (class A) was clearly higher in the group fed BI than that of control worms (Fig. 2).

We also found that autofluorescence of lipofuscin, a lipid peroxidation product, was significantly lower among worms fed BI than that of control worms (Fig. 3a-c). For this 
1 reason, the protein carbonyl content, which is a general biomarker of protein oxidation, was

2 also measured in treated and control worms. The abundance of protein carbonyls was greater

3 in the extracts from control worms than those from worms fed BI (Fig. 4a, b).

As mitochondria are involved in the oxidation of biomolecules, and because both

5 lipofuscin and protein carbonyls were increased in control worms compared to the levels in

6 worms fed BI, the copy number of mitochondria in 3- and 7-day-old worms was measured

7 using real-time PCR. However, no significant differences in the copy number of the $c t b-1$

8 gene, which resides on the mitochondrial chromosome, were detected between worms fed OP

9 and those fed BI, irrespective of their ages (data not shown), indicating that the number of 10 mitochondria was not affected by BI feeding.

Body and brood sizes

To examine whether the prolongevity effects of BI were a result of caloric reduction, the body and brood sizes of nematodes fed BI were compared with those of control worms fed OP. The feeding of nematodes BI did not alter the growth rate or body size of worms (Fig. 5). Although caloric restriction reportedly extends the lifespan of nematodes, (Bishop and Guarente, 2007), here, the mixed feeding of BI and OP not only extended the lifespan of $C$. elegans, but also increased the brood size of worms (Fig. 6).

21 Stress resistance assays

23 We previously reported that lactobacilli and bifidobacteria confer tolerance to bacterial 24 infections in C. elegans (Ikeda et al., 2007; Komura et al., 2010). Here, the effects of feeding worms BI on resistance to physical and chemical stresses were examined. BI treatment 
1 failed to improve the survival of worms against exposure to 1.0 and $2.5 \mathrm{mM}$ paraquat, or

$2 \quad 35^{\circ} \mathrm{C}$ heat stress (data not shown). In addition, when 7-day-old worms were maintained in M9

3 buffer containing either 0.8 or $2.0 \mathrm{mM}$ hydrogen peroxide, the worms fed BI were more

4 vulnerable than the controls, as exhibited by the shorter survival time of the former (Fig. 7a

5 and $7 b$ ). Notably, BI-treated 3-day-old adult worms were killed more quickly on exposure to

6 hydrogen peroxide than 7-day-old nematodes.

7

8 Prolongevity-associated components of bifidobacteria

9

To determine whether the lifespan-extending factor of bifidobacteria is located in the cell wall, worms were fed the cell walls or protoplasts, which were negative for peptidoglycan, of BI. However, the lifespan of worms was prolonged similarly by cell walls and protoplasts (Fig. 8a and $8 b)$.

Prolongevity effects in mutants

16

The PMK-1 (p38 mitogen-activated protein kinase; p38 MAPK) and DAF-2/DAF-16 insulin-like signaling pathways contribute to host defense against pathogens. To investigate whether these two pathways are involved in the prolongevity effects of BI, the lifespan of several C. elegans loss-of-function mutants fed BI was compared with that of control worms fed OP (Figs. 9 and 10). BI failed to prolong the lifespans of pmk-1 and skn-1 mutants. As these results suggested the importance of the p38 MAPK pathway for the prolongevity effect of BI, mutants of genes (tol-1, tir-1, jnk-1, and $v h p-1)$ that are possibly associated with the p38 MAPK pathway were also examined. BI prolonged the mean lifespans of the tol-1, tir-1, and jnk-1 mutants by $113 \%$ to $155 \%$, whereas that of the $v h p-1$ mutant was not extended by 
1 BI treatment. Concerning the insulin/IGF-1 signaling (IIS) pathway, BI treatment extended

2 the lifespan of the daf-16 C. elegans mutant; however, the lifespan of the daf-2 mutant was

3 not markedly affected. A mutant of aak-2, which encodes the low-energy sensing

4 AMP-activated protein kinase AMPK, was also used to investigate the contribution of caloric

5 restriction to the prolongevity effect of BI. Upon BI feeding, the lifespan of the aak-2 mutant

6 was clearly extended.

7

8

9

10

11

12

Based on the observed longevity of Bulgarians who consumed large quantities of yogurt, Metchnikoff hypothesized over a century ago that lactobacilli are important for human health (Metchnikoff, 1907). Previously, we demonstrated that lactobacilli and bifidobacteria modify the host defense and prolong the lifespan of nematodes (Ikeda et al., 2007; Komura et al., 2012; Komura et al., 2010); however, the mechanism by which these probiotic bacteria affect the longevity of bacteriovorus nematodes remained unclear. In the present study, we have shown that bifidobacteria can increase the average lifespan of $C$. elegans in a dose-dependent manner due to components present in the bacterial cell wall. Our findings suggest that $C$. elegans is a useful model for evaluating the potential of probiotic bacteria as biological response modifiers in higher eukaryotes, because LAB, including bifidobacteria, have developed symbiotic relationships with a variety of animals prior to the appearance of humans on earth.

When BI mixed with OP was fed to C. elegans, the lifespan of worms was extended in a BI dose-dependent manner. The worms fed BI clearly displayed more locomotion than age-matched control worms. The typical protein modification associated with oxidative stress 
1 is the addition of carbonyl groups to amino acid side chains. Here, the accumulation of

2 lipofuscin, a marker of aging, and the levels of protein carbonyls were lowest in worms fed

3 bifidobacteria. The total amount of ROS leaking from mitochondria is thought to be small and

4 result in lower lipofuscin and protein carbonyl levels in BI-fed worms (Cannizzo et al., 2011;

5 Pincus and Slack, 2010). Although the concept that caloric restriction upregulates

6 mitochondrial biogenesis and may confer anti-aging effects is generally accepted (Guarente,

7 2008), we did not observe a difference in the number of mitochondria in response to BI 8 feeding.

9 Caloric restriction is well recognized as a method to extend longevity and has been shown to slow aging not only in numerous non-mammalian taxa, but also in mammals (Ingram et al., 2006). However, it is unlikely that bifidobacteria have lower caloric energy or are simply more indigestible than OP because the growth curves of nematodes were similar regardless of the amount of BI mixed with OP. Furthermore, the BI-fed nematodes produced more offspring than the control worms, a finding that is in contrast to a study by Bishop and Guarente (2007), who showed that although dietary restriction can extend the life of worms, their brood sizes are smaller than worms fed a normal diet (Bishop and Guarente, 2007).

We previously observed that $\mathrm{LAB}$ and bifidobacteria suppress the age-associated increase in sensitivity of $C$. elegans to bacterial infection (Ikeda et al., 2007; Komura et al., 2010). Consequently, we anticipated that the host defense mechanisms to a variety of stresses would be enhanced in worms fed bifidobacteria. The lower lipofuscin and protein carbonyl levels in BI-fed worms were taken as evidence of enhanced antioxidant systems. However, the BI-induced prolongevity was unlikely due to the non-specific enhancement of host defense systems, as the worms were as vulnerable to heat and paraquat as the control worms. In addition, the BI-fed worms had lower resistance to the oxidative stress induced by hydrogen peroxide than the control worms; however, this finding may reflect the degree of aging of 
1 BI-fed worms, as 7-day-old BI-fed worms exhibited similar susceptibility to hydrogen

2 peroxide as 3-day-old control worms. If age was not an influential factor, this phenomenon

3 would be consistent with the trade-off of host defense reported by Marsh et al. (2011), who

4 observed that deletion of the protist-type lysozyme LYS-7 in nematodes enhanced tolerance

5 to Salmonella, but increased susceptibility to Cryptococcus neoformans.

6 BI failed to prolong the lifespan of the C. elegans pmk-1 and skn-1 mutants, suggesting 7 that the p38 MAPK pathway contributes to the prolongevity effect of bifidobacteria in nematodes (Fig. 11). BI appears to promote the induction of phase 2 detoxification enzymes

in C. elegans through activation of SKN-1, an ortholog of mammalian Nrf transcription factors (Inoue et al., 2005). The p38 MAPK pathway elicits an immune response distinct from that associated with DAF-16 (Troemel et al., 2006) via the activation of $s k n-1$ (Inoue et al., 2005; Van der Hoeven et al., 2011). Recently, Lactobacillus acidophilus was also reported to function as an immunomodifier in $C$. elegans via signals transmitted by the p38 MAPK pathway (Kim and Mylonakis, 2012). As we found that BI prolonged the lifespans of tol-1 and tir-1 mutants of $C$. elegans, the pattern recognition system in innate immunity is not likely involved in the activation of p38 MAPK by BI. PMK-1 is inactivated by the MAPK phosphatase VHP-1, whose suppression reportedly increases PMK-1 phosphorylation and increases resistance to pathogens (Kim et al., 2004). It is likely that the prolongevity effect of BI was reduced in the $v h p-1$ mutant because $p m k-1$ was upregulated irrespective of $\mathrm{BI}$ treatment in the absence of VHP-1-mediated suppression. It also seems likely that BI induces

21 prolongevity in C. elegans via suppression of $v h p-1$, a speculation that is consistent with the 22 fact that the BI factor associated with prolongevity would not interact with the MAPK phosphatase VHP-1 in the $v h p-1$ mutant. In addition, the comparatively shorter lifespan of the vhp-1 mutants is consistent with the adverse effects of excessive SKN-1 activity in C. elegans

(Papp et al., 2012). 
Although C. elegans is bacteriophagous, bacteria typically consumed as a food source can

2 be pathogenic to older nematodes (Garigan et al., 2002). Therefore, the activation of host defense systems via pmk-1 through the ingestion of BI may have contributed to the observed prolongevity effects. However, the increased lifespan of the BI-fed worms is unlikely explained solely by the upregulation of antimicrobial defense systems, as these worms lived longer than those fed heat-killed OP (Ikeda et al., 2007). As no differences in the number of pathogens recovered after infection with Salmonella or Legionella were detected between BIand OP-fed worms in our previous studies (Ikeda et al., 2007; Komura et al., 2010), it is also unlikely that the enhanced survival of worms observed here was due to increased expression of antibacterial factors induced by BI. Through experiments using Drosophila melanogaster as a model host for Salmonella infection, Shinzawa et al. (2009) also reported that p38 MAPK is involved in host tolerance to this pathogen. As SKN-1 appears to be involved in damage control and stress resistance (Oliveira et al., 2009), endogenous antioxidant systems upregulated via activated SKN-1 may account for the enhanced tolerance to pathogens, extension of lifespan, and reduced accumulation of lipofuscin and protein carbonyls in worms fed BI. Notably, however, the activity of these antioxidant systems was insufficient to protect cells from exogenous oxidants. Oliveira et al. (2009) reported that most genes controlled by SKN-1 under normal conditions are not upregulated in response to chemical stress caused by sodium arsenite or butyl hydrogen peroxide; thus, SKN-1 appears to act together with other regulators involved in specialized responses to exogenous stresses. Future experiments comparing transcriptional changes in pmk-1 mutant and wild-type nematodes fed BI are expected to reveal which genes under the control of pmk-1 contribute to prolongevity in $C$. elegans.

24 Downstream of the insulin/IGF-1 signaling (IIS) pathway, which has a conserved role in modulating lifespan, the forkhead family transcription factor DAF-16 regulates genes that 
1 promote stress resistance and extend the lifespan of nematodes (Lee et al., 2003). Heat-shock

2 transcription factor (HSF)-1 is one such gene under the regulation of DAF-16 also plays an 3 important role for host defense (McColl et al., 2010; Singh and Aballay, 2006). Oh et al. 4 (2005) found that the c-Jun N-terminal kinase (JNK) family, a subgroup of the MAPK 5 superfamily, is a positive regulator of DAF-16. As JNK is part of a signal transduction 6 cascade in mammals that is activated by cytokines, including TNF and IL-1, it is possible that 7 bifidobacteria enhance host defense systems and extend the lifespan of nematodes by activating DAF-16 via the JNK pathway. However, we found that neither the $d a f-16$ nor $j n k-1$ genes were necessary for the prolongevity effect of BI in C. elegans. Although worms with activated DAF-16 should have upregulated sod-3 expression and exhibit resistance to heat or chemical oxidants (Murphy et al., 2003), BI feeding did not increase the resistance of worms to either heat or oxidants in the present study. As the signal induction systems involved in host defense should respond to stressors and modulate stress responses to minimize damage to the host, it is logical that the prolongevity induced by BI feeding does not necessarily lead to nonspecific resistance to chemical oxidants. [

BI failed to prolong the lifespan of the $d a f-2$ mutant. At first glance, this result appears contradictory to the prolongevity effects of BI observed for the daf-16 mutant; however, this discrepancy is likely because not only the p38 MAPK, but also the IIS pathway, for which DAF-2 is the only insulin/IGF-1 receptor, regulates SKN-1, which modulates both oxidative and xenobiotic stress responses in C. elegans (Inoue et al., 2005; Tullet et al., 2008). It is 21 possible that the $s k n-1$ gene was no longer under control of DAF-2 signaling and was sufficiently activated in the $d a f-2$ mutant to enhance host resistance, with the addition of BI having little additional effect on the activation of SKN-1. Once the $s k n-1$ gene is no longer suppressed by the IIS pathway, SKN-1 seems to act against the daf-28 and ins-39 genes, which encode insulin-like substances, suggesting that SKN-1 could cause a positive feedback, 
1 thereby mitigating the enhancement of SKN-1 by BI (Okuyama et al., 2010). Although the

2 lack of the prolongevity effect of $\mathrm{BI}$ in $d a f-2$ is indicative of caloric restriction, dietary 3 restriction has been shown to extend the lifespan of daf-2 C. elegans mutants (Bishop and

4 Guarente, 2007). Furthermore, successful prolongation of the lifespan of the aak-2 mutant fed 5 BI also negates the possibility that BI induces longevity via caloric restriction, as AAK-2 6 plays an important role in prolongevity in response to caloric restriction (Apfeld et al., 2004). The cell walls of LAB are thought to be primarily responsible for immunostimulation, and differences in the cell wall composition of probiotic organisms may account for the various levels of immune activation observed in hosts (Rutherfurd-Markwick and Gill, 2004). Here, the lifespan of worms fed OP together with the cell wall fraction of BI was clearly extended. Hence, we assumed that the protoplast of BI does not induce longevity in worms. Surprisingly, however, the feeding of BI protoplasts to $C$. elegans extended the mean lifespan of worms. Among the possible component(s) that are present in both protoplasts and cell walls, we speculate that lipoteichoic acid might be the responsible factor, as it may have remained on the bacterial cell membrane even after the formation of protoplasts by enzymatic treatment.

In conclusion, the findings from the present study suggest that bifidobacteria extend the lifespan of $C$. elegans, presumably via modulation of the p38 MAPK pathway, but not via caloric restriction. Notably, we found that the cell wall of bifidobacteria plays a key role in increasing the longevity of nematodes. After we previously reported that C. elegans is an appropriate model to screen for useful probiotic strains, two groups succeeded in selecting 21 potentially therapeutic strains using this model (Lee et al., 2011; Wang et al., 2011). However, time-consuming lifespan analyses need to be performed with these strains, as the mechanisms involved in the prolongevity effects remain to be clarified. Our present findings suggest that a gene involved in the p38 MAPK pathway can be utilized as an indicator of prolongevity induced by bifidobacteria. If this C. elegans gene is identified and labeled with GFP, it would 
1 be possible to screen for probiotic strains by observing GFP expression in worms after the 2 ingestion of candidate bacteria.

3 


\section{Acknowledgements}

2

3 We would like to express our sincere thanks to Bch. Yuji Onimaru at the Graduate School of

4 Human Life Science, Osaka City University, for his technical support for the quantification of

5 mitochondria. This study was supported in part by a Grant-in-aid for Scientific Research C

6 (No. 23617017) from the Japan Society for the Promotion of Science, a Grant of Adaptable

7 and Seamless Technology Transfer Program through target-driven R\&D of the Japan Science

8 and Technology Agency, a Grant from Research Fellowships of the Japan Society for the

9 Promotion of Science for Young Scientist (to T.K.), and a Grant of the Osaka City University

10 Graduate School of Human Life Science in 2010 and 2011. The nematodes used in this study

11 were kindly provided by the Caenorhabditis Genetics Center, which is funded by the NIH

12 National Center for Research Resources (NCRR). 


\section{References}

Anyanful A, Dolan-Livengood JM, Lewis T, Sheth S, Dezalia MN, Sherman MA, Kalman LV, Benian GM, Kalman D (2005) Paralysis and killing of Caenorhabditis elegans by enteropathogenic Escherichia coli requires the bacterial tryptophanase gene. Mol Microbiol 57:988-1007

Apfeld J, O'Connor G, McDonagh T, DiStefano PS, Curtis R (2004) The AMP-activated protein kinase AAK-2 links energy levels and insulin-like signals to lifespan in $C$. elegans. Genes Dev 18:3004-3009

Austad SN (2012) AGEING Mixed results for dieting monkeys. Nature 489:210-211

Bishop NA, Guarente L (2007) Two neurons mediate diet-restriction-induced longevity in $C$. elegans. Nature 447:545-549.

Bogden JD, Louria DB (2004) Nutrition and immunity in the elderly. In: Hughes DA, Gail

Finch CE, Ruvkun G (2001) The genetics of aging. Annu Rev Genomics Hum Genet

Effros RB, Walford RL, Weindruch R, Mitcheltree C (1991) Influences of dietary restriction on immunity to influenza in aged mice. J Gerontol 46:B142-147 $2: 435-462$

Fulop T, Larbi A, Hirokawa K, Mocchegiani E, Lesourd B, Castle S, Wikby A, Franceschi C, 
1 Garigan D, Hsu AL, Fraser AG, Kamath RS, Ahringer J, Kenyon C (2002) Genetic analysis of tissue aging in Caenorhabditis elegans: a role for heat-shock factor and bacterial proliferation. Genetics 161:1101-1112

Gregersen T (1978) Rapid method for distinction of gram-negative from gram-positive bacteria. Eur J Appl Microbiol Biotechnol 5:123-127

Grubeck-Loebenstein B (1997) Changes in the aging immune system. Biologicals 25:205-208

Gruber J, Ng LF, Fong S, Wong YT, Koh SA, Chen CB, Shui G, Cheong WF, Schaffer S, Wenk MR, Halliwell B (2011) Mitochondrial changes in ageing Caenorhabditis elegans--what do we learn from superoxide dismutase knockouts? PLoS ONE 6:e19444

Guarente L (2008) Mitochondria--a nexus for aging, calorie restriction, and sirtuins? Cell $132: 171-176$

Hayek MG, Taylor SF, Bender BS, Han SN, Meydani M, Smith DE, Eghtesada S, Meydani SN (1997) Vitamin E supplementation decreases lung virus titers in mice infected with influenza. J Infect Dis 176:273-276

Hosono R, Sato Y, Aizawa SI, Mitsui Y (1980) Age-dependent changes in mobility and separation of the nematode Caenorhabditis elegans. Exp Gerontol 15:285-289

Hsin H, Kenyon C (1999) Signals from the reproductive system regulate the lifespan of $C$. elegans. Nature 399:362-366

Ikeda T, Yasui C, Hoshino K, Arikawa K, Nishikawa Y (2007) Influence of lactic acid bacteria on longevity of Caenorhabditis elegans and host defense against Salmonella entetica serovar Enteritidis. Appl Environ Microbiol 73:6404-6409

Ingram DK, Zhu M, Mamczarz J, Zou S, Lane MA, Roth GS, deCabo R (2006) Calorie restriction mimetics: an emerging research field. Aging Cell 5:97-108

Inoue H, Hisamoto N, An JH, Oliveira RP, Nishida E, Blackwell TK, Matsumoto K (2005) The C. elegans p38 MAPK pathway regulates nuclear localization of the transcription 
factor SKN-1 in oxidative stress response. Genes Dev 19:2278-2283

2 Kim DH, Liberati NT, Mizuno T, Inoue H, Hisamoto N, Matsumoto K, Ausubel FM (2004) Integration of Caenorhabditis elegans MAPK pathways mediating immunity and stress resistance by MEK-1 MAPK kinase and VHP-1 MAPK phosphatase. Proc Natl Acad Sci U S A 101:10990-10994

Kim Y, Mylonakis E (2012) Caenorhabditis elegans immune conditioning with the probiotic bacterium Lactobacillus acidophilus strain NCFM enhances Gram-positive immune responses. Infect Immun 80:2500-2508

Komura T, Yasui C, Miyamoto H, Nishikawa Y (2010) Caenorhabditis elegans as an alternative model host for Legionella pneumophila and the protective effects of Bifidobacterium infantis. Appl Environ Microbiol 76:4105-4108

Komura T, Ikeda T, Hoshino K, Shibamura A, Nishikawa Y (2012) Caenorhabditis elegans as an Alternative Model to Study Senescence of Host Defense and the Prevention by Immunonutrition. Adv Exp Med Biol 710:19-27

Lee-Wickner LJ, Chassy BM (1984) Production and regeneration of Lactobacillus casei protoplasts. Appl Environ Microbiol 48:994-1000

Lee J, Yun HS, Cho KW, Oh S, Kim SH, Chun T, Kim B, Whang KY (2011) Evaluation of probiotic characteristics of newly isolated Lactobacillus spp.: immune modulation and longevity. Int J Food Microbiol 148:80-86

Lee SS, Kennedy S, Tolonen AC, Ruvkun G (2003) DAF-16 target genes that control $C$. elegans life-span and metabolism. Science 300:644-647

Marsh EK, van den Berg MC, May RC (2011) A two-gene balance regulates Salmonella typhimurium tolerance in the nematode Caenorhabditis elegans. PLoS ONE 6:e16839

McColl G, Rogers AN, Alavez S, Hubbard AE, Melov S, Link CD, Bush AI, Kapahi P, Lithgow GJ (2010) Insulin-like Signaling Determines Survival during Stress via 
2 Metchnikoff E (1907) The Prolongation of Life. Heinemann, London

3 Miller E, Gay N (1997) Effect of age on outcome and epidemiology of infectious diseases. Biologicals 25:137-142

Moulias R, Devillechabrolle A, Lesourd B, Proust J, Marescot MR, Doumerc S, Favre Berrone M, Congy F, Wang A (1985) Respective roles of immune and nutritional factors in the priming of the immune response in the elderly. Mech Ageing Dev 31:123-137

Murphy CT, McCarroll SA, Bargmann CI, Fraser A, Kamath RS, Ahringer J, Li H, Kenyon C (2003) Genes that act downstream of DAF-16 to influence the lifespan of Caenorhabditis elegans. Nature 424:277-283

Naidu AS, Bidlack WR, Clemens RA (1999) Probiotic spectra of lactic acid bacteria (LAB). Crit Rev Food Sci Nutr 39:13-126

Oh SW, Mukhopadhyay A, Svrzikapa N, Jiang F, Davis RJ, Tissenbaum HA (2005) JNK regulates lifespan in Caenorhabditis elegans by modulating nuclear translocation of forkhead transcription factor/DAF-16. Proc Natl Acad Sci U S A 102:4494-4499

Okuyama T, Inoue H, Ookuma S, Satoh T, Kano K, Honjoh S, Hisamoto N, Matsumoto K, Nishida E (2010) The ERK-MAPK pathway regulates longevity through SKN-1 and insulin-like signaling in Caenorhabditis elegans. J Biol Chem 285:30274-30281

Oliveira RP, Porter Abate J, Dilks K, Landis J, Ashraf J, Murphy CT, Blackwell TK (2009) Condition-adapted stress and longevity gene regulation by Caenorhabditis elegans SKN-1/Nrf. Aging Cell 8:524-541

Ottaviani E, Ventura N, Mandrioli M, Candela M, Franchini A, Franceschi C (2011) Gut microbiota as a candidate for lifespan extension: an ecological/evolutionary perspective targeted on living organisms as metaorganisms. Biogerontology 12:599-609

Papp D, Csermely P, Soti C (2012) A Role for SKN-1/Nrf in Pathogen Resistance and 
2 Pawelec G, Larbi A (2008) Immunity and ageing in man: Annual review 2006/2007. Experimental Gerontology 43:34-38

Pincus Z, Slack FJ (2010) Developmental biomarkers of aging in Caenorhabditis elegans. Developmental Dynamics 239:1306-1314

Pop-Vicas A, Gravenstein S (2011) Influenza in the Elderly - A Mini-Review. Gerontology 7

Reinke SN, Hu X, Sykes BD, Lemire BD (2010) Caenorhabditis elegans diet significantly affects metabolic profile, mitochondrial DNA levels, lifespan and brood size. Mol Genet Metab 100:274-282

Riddle DL, Blumenthal T, Meyer BJ, Priess JR (Eds.), 1997. C. ELEGANS II. Cold Spring Harbor Laboratory Press, Cold Spring Harbor.

Ristow M, Schmeisser S (2011) Extending life span by increasing oxidative stress. Free Radic Biol Med 51:327-336

Rutherfurd-Markwick KJ, Gill HS (2004) Probiotics and immunomodulation. In: Hughes DA, Darlington LG, Bendich A (eds.) Diet and human immune function. Humana Press, Totowa, pp 327-344

Shinzawa N, Nelson B, Aonuma H, Okado K, Fukumoto S, Miura M, Kanuka H (2009) p38 MAPK-dependentphagocytic encapsulation confers infection tolerance in Drosophila. Cell Host Microbe 6:244-252

Singh V, Aballay A (2006) Heat-shock transcription factor (HSF)-1 pathway required for Caenorhabditis elegans immunity. Proc Natl Acad Sci U S A 103:13092-13097

Sligl WI, Majumdar SR (2011) How important is age in defining the prognosis of patients with community-acquired pneumonia? Curr Opin Infect Dis 24:142-147 
approach. Oxford University Press, New York, pp 51-67

2 Sulston J, Hodgkin J (1988) Methods. In: Wood WB, researchers TcoCe (eds.) The nematode Caenorhabditis elegans. Cold Spring Harbor Laboratory Press, New York, pp 587-606

Tejada-Simon MV, Pestka JJ (1999) Proinflammatory cytokine and nitric oxide induction in murine macrophages by cell wall cytoplasmic extracts of lactic acid bacteria. J Food Prot $62: 1435-1444$

Troemel ER, Chu SW, Reinke V, Lee SS, Ausubel FM, Kim DH (2006) p38 MAPK regulates expression of immune response genes and contributes to longevity in C. elegans. PLoS Genet 2:1725-1739

Tullet JM, Hertweck M, An JH, Baker J, Hwang JY, Liu S, Oliveira RP, Baumeister R, Blackwell TK (2008) Direct inhibition of the longevity-promoting factor SKN-1 by insulin-like signaling in C. elegans. Cell 132:1025-1038

Van der Hoeven R, McCallum KC, Cruz MR, Garsin DA (2011) Ce-Duox1/BLI-3 generated mutants of Caenorhabditis elegans. Genetics 177:2063-2074

Wang C, Wang J, Gong J, Yu H, Pacan JC, Niu Z, Si W, Sabour PM (2011) Use of Caenorhabditis elegans for preselecting Lactobacillus isolates to control Salmonella Typhimurium. J Food Prot 74:86-93

Wu D, Rea SL, Yashin AI, Johnson TE (2006) Visualizing hidden heterogeneity in isogenic populations of C. elegans. Exp Gerontol 41:261-270

Yang W, Li J, Hekimi S (2007) A Measurable increase in oxidative damage due to reduction in superoxide detoxification fails to shorten the life span of long-lived mitochondrial 


\section{Figure legends}

Fig. 1. Survival curves of $C$. elegans fed OP mixed with BI at different ratios as compared with the lifespan of control worms fed only OP. Each plate contained $10 \mathrm{mg}$ (wet weight) of bacteria. Worms were 3-days-old on Day 0. The table summarizes the data obtained from all experiments, and the curve was drawn based on the results of a representative experiment.

Fig. 2. Locomotory activity of C. elegans fed BI. Young adult worms fed OP for 2 days after hatching were transferred to plates containing $10 \mathrm{mg}$ of either OP or BI on the surface. Worms were 3-days-old on Day 0. Animals were classified into four classes based on their locomotion: class A, robust, coordinated sinusoidal locomotion (blue bars); class B, uncoordinated and/or sluggish movement (yellow bars); class $\mathrm{C}$, no forward or backward movement, but head movements or shuddering in response to prodding (red bars); and class D, dead animals (black bars). The rates of each class at the indicated time point are indicated.

Fig. 3. Lipofuscin accumulation in the intestine of nematodes. (a) Intestinal autofluorescence from lipofuscin in age-synchronized worms fed OP was apparent on day 14, but was weaker in worms fed bifidobacteria (b). (c) Fluorescence on days 14, 16, and 18 of adulthood was quantified using ImageJ software to determine the lipofuscin levels. Each bar represents the average values of ten worms, except for the bar corresponding day 18 , for which six OP-fed worms. The bar graph depicts the percent difference of the mean value in arbitrary units relative to that of control worms fed OP on day 14. Lipofuscin fluorescence was weaker among worms fed BI compared with control worms fed OP. ** indicates a statistically significant difference from control worms fed OP at a $p$ value of $<0.01$. Error bars represent the SE. 
Fig. 4. Influence of BI-feeding on protein oxidation evaluated using an OxyBlot kit. Non-derivatized proteins were used as negative controls for each sample. (A) Proteins recovered from lysed worms fed BI or OP were labeled with DNP solution (Oxyblot) to show the presence of protein carbonyls and were separated on SDS-PAGE gels (lanes OP and BI). Derivatization-control proteins were also included (lanes BI-neg and OP-neg). After electrophoresis, the proteins were transferred to a PVDF membrane and visualized using a chemiluminescence method. The membrane was re-probed with anti-actin antibody to correct for loading variation. (B) Band densities were quantified to determine the protein carbonyl levels using ImageJ software. The bar graph depicts the percent difference of the mean value of five independent experiments (error bars represent the S.E.) in arbitrary units relative to that of control worms fed OP. Actin or coloration of CBB-stained bands was used to compensate for loading variation. * indicates a statistically significant difference from control worms fed OP at a $p$ value of 0.037 .

Fig. 5. Growth curve of worms fed OP mixed with BI at ratios of 3:7 or 1:4. Images of adult nematodes were taken with a digital microscope, and the area of the worm's projection was measured and used as an index of body size. The body sizes of the worms fed BI at all ratios were similar to those of the control worms fed only OP. No value reached the significance limit of $p<0.05$ compared with control worms. All results are presented as means \pm SD.

Fig. 6. Brood size of BI-fed worms. Total brood size was determined from 30 animals. * indicates statistical significance at $p<0.05$ compared to the control worms fed OP only. Error bars represent the SE. 
Fig. 7. Influence of BI-feeding on susceptibility of worms to oxidative stress caused by hydrogen peroxide. Worms were fed BI for 4 days beginning from 3 days of age and then incubated with (a) $0.8 \mathrm{mM}$ or (b) $2 \mathrm{mM}$ hydrogen peroxide. ${ }^{\S} p=1.1 \mathrm{E}-10,{ }^{\dagger} p=1.73 \mathrm{E}-13,{ }^{\Psi} p$ $=0.002176$, and $\$ p=2.83 \mathrm{E}-06$, as compared to the control worms exposed to hydrogen peroxide from 7 days of age.

Fig. 8. Survival curves of $C$. elegans fed the protoplasts or cell walls of bifidobacteria mixed with OP. Adult worms fed OP for 3 days after hatching were placed onto plates containing OP ( $2 \mathrm{mg}$ ) mixed with the cell wall $(8 \mathrm{mg}$ ) or protoplasts of BI as a food source. Worms were 3 days of age at day 0 . The lifespan of worms was prolonged similarly by cell walls (a) and protoplasts (b). The table shows the data obtained from all experiments, and the curves were drawn based on representative experiments.

Fig. 9. Effects of bifidobacteria on the lifespan of C. elegans mutants. Survival curves of (a) pmk-1(km25), (b) skn-1(zu67), (c) vhp-1(sa366), (d) tol-1(nr2033), and (e) tir-1(ok1052) mutant hermaphrodites fed with or without BI from 3 days of age are shown. The worms were 3 days of age at day 0 . The table summarizes the data obtained from all experiments, and the curves were drawn based on representative experiments.

Fig. 10. Effects of bifidobacteria on the lifespan of C. elegans mutants. Survival curves of (a) daf-16(mu86), (b) daf-2(e1370), (c) jnk-1(gk-7), and (d) aak-2(ok524) mutant hermaphrodites fed with or without BI from 3 days of age are shown. The worms were 3 days of age at day 0; however, the daf-2 mutants were 7 days of age at day 0 due to their slow growth. The tables summarize the data of all experiments, and the curves were drawn based on a representative experiment. 
Fig. 11. Pathways predicted to be involved in the prolongevity effect of bifidobacteria. Red lines indicate signals that upregulate or activate genes, and black lines indicate signals involved in suppression. Established pathways are shown by solid lines. Dotted lines indicate signals or genes whose contribution to the prolongevity effects of BI was estimated to be minimal. Dashed lines suggest hypothetical pathways that remain to be elucidated. 


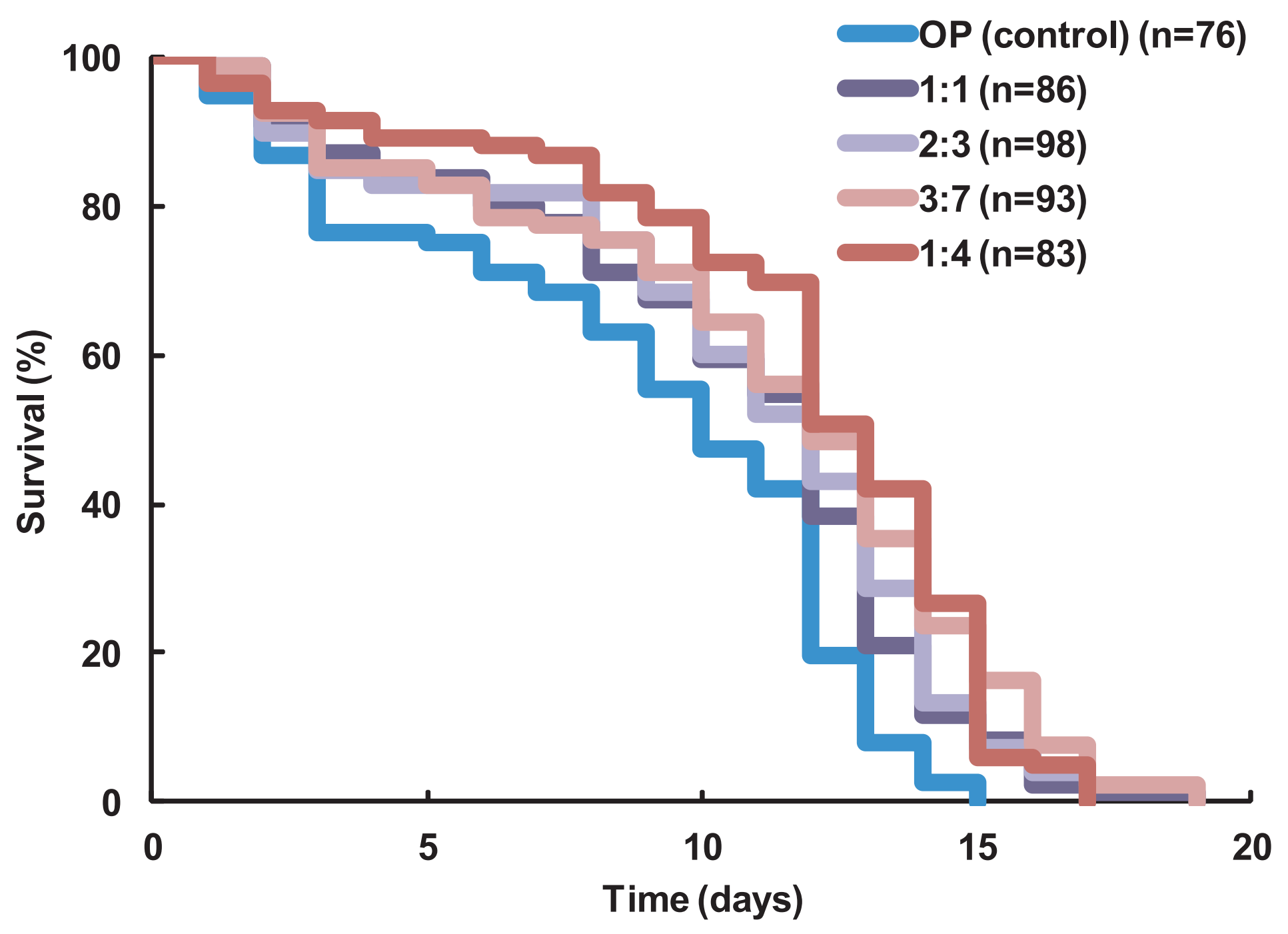




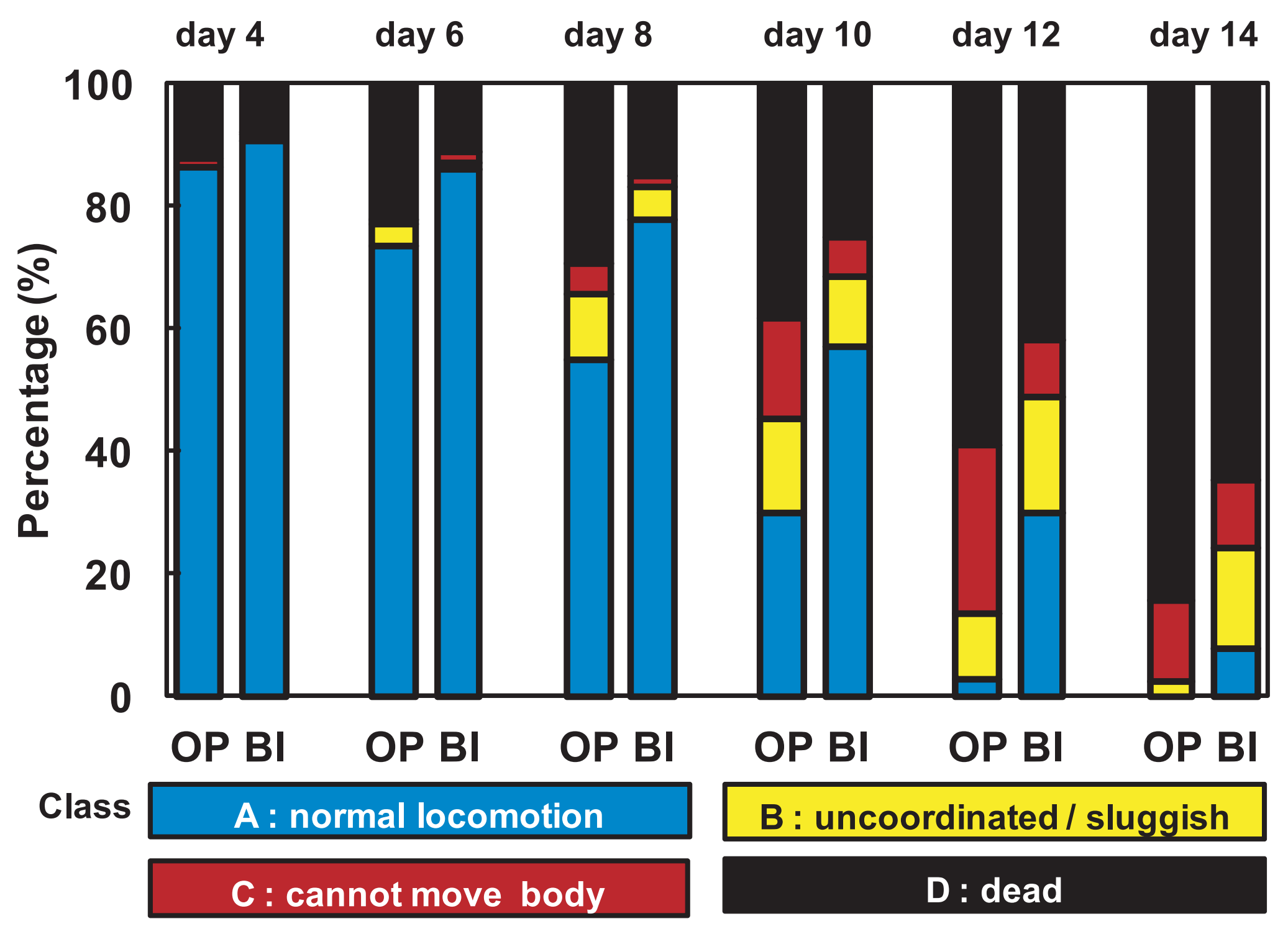



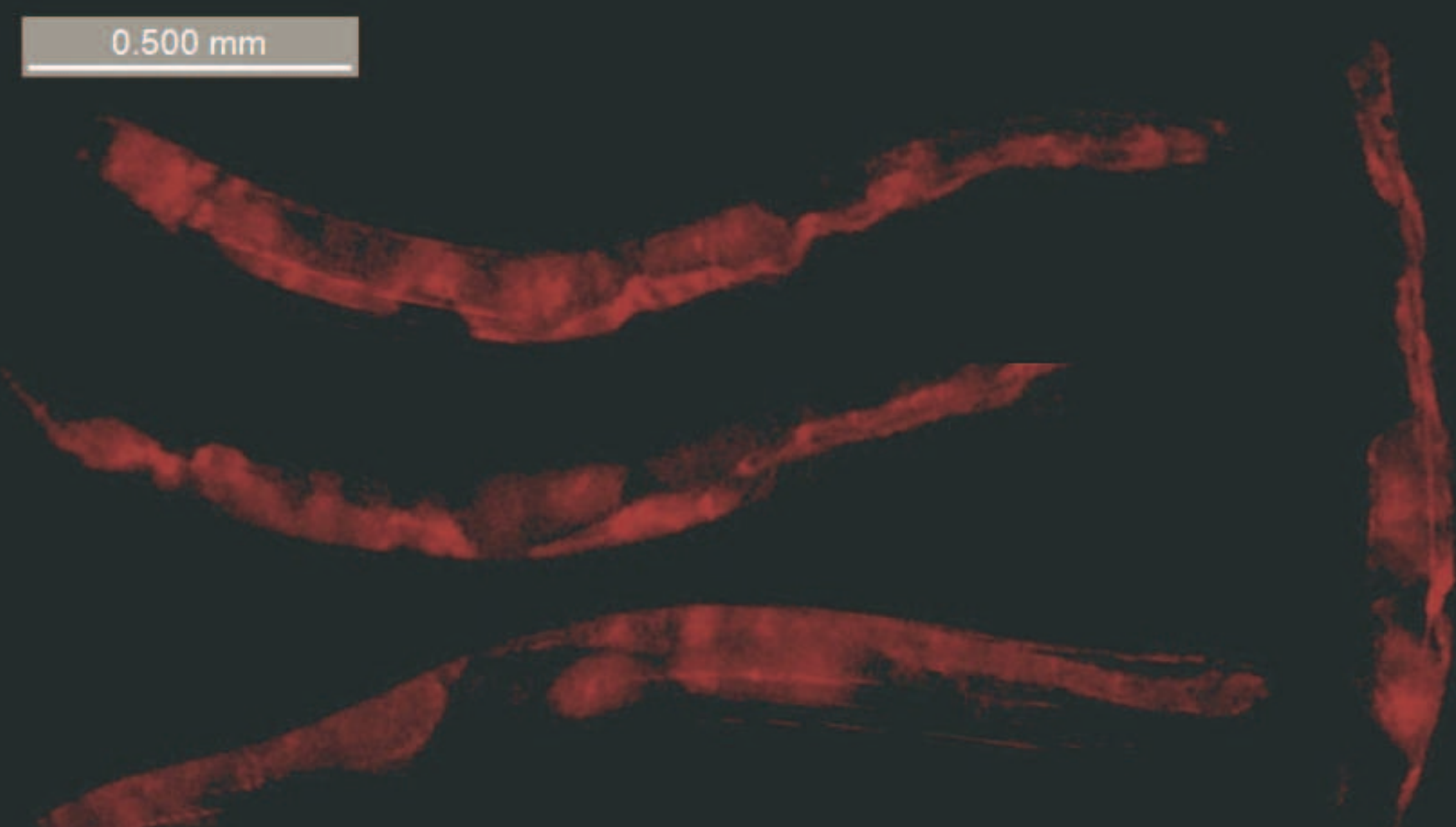


$$
0.500 \mathrm{~mm}
$$




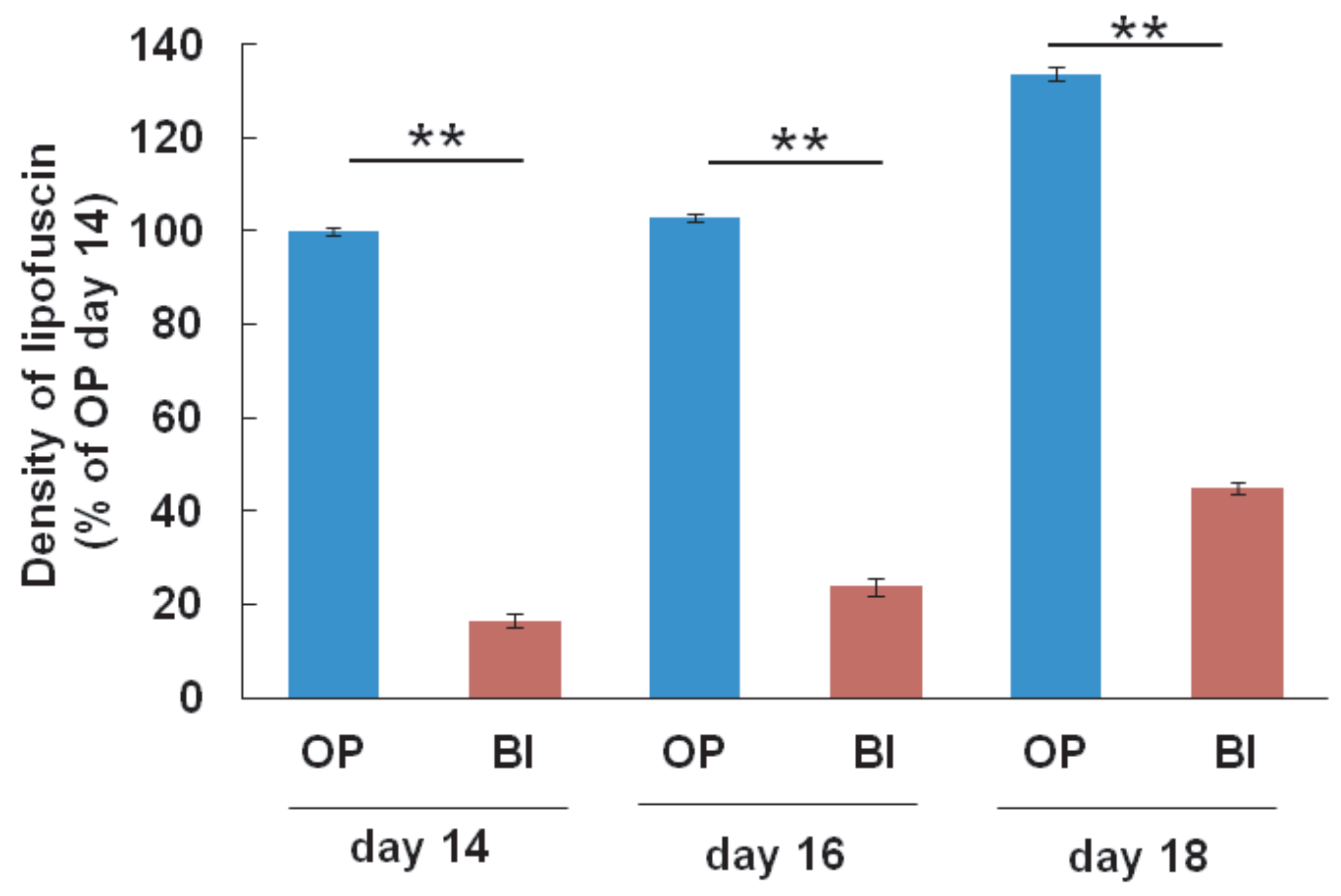




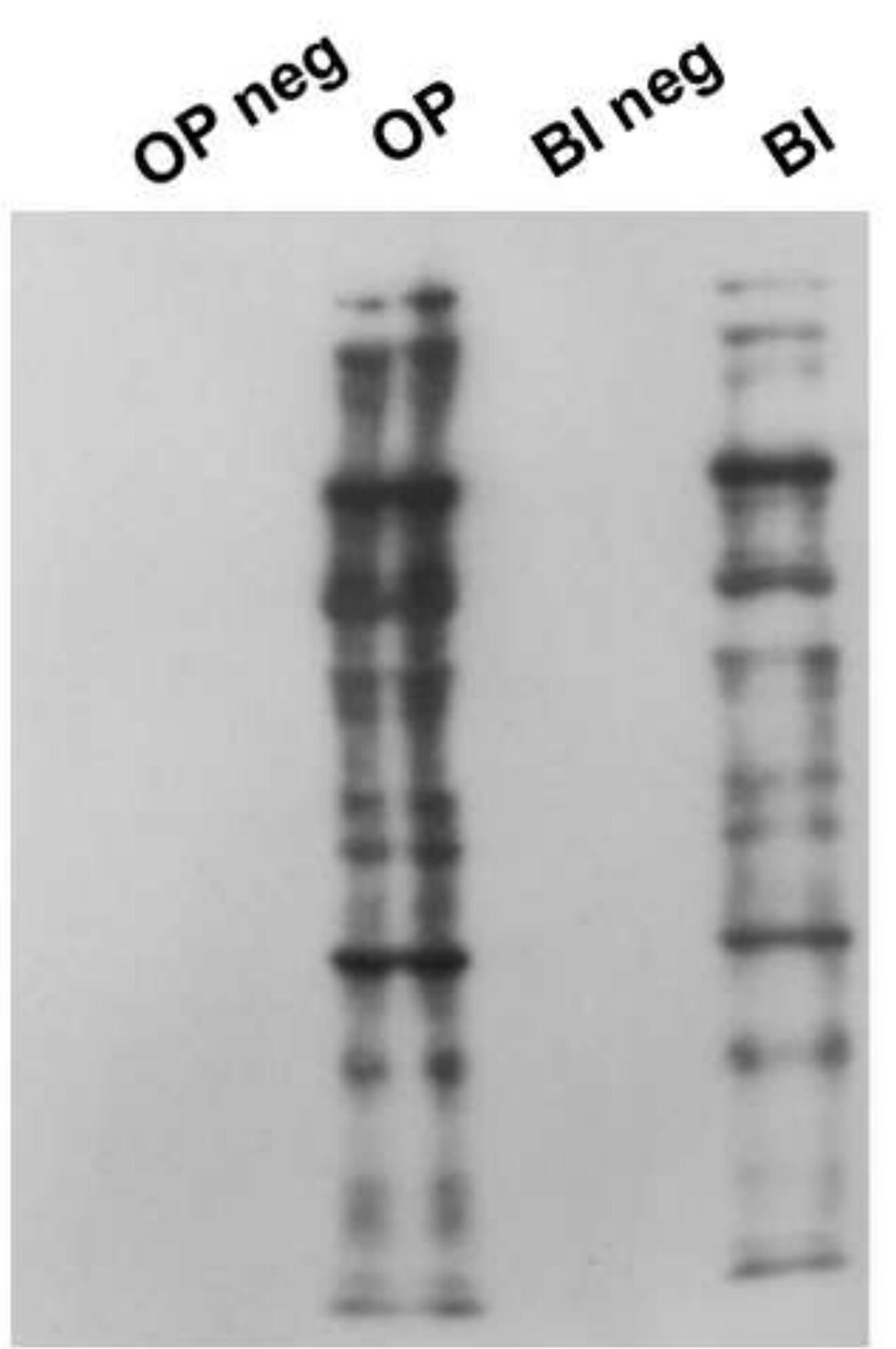

Actin 


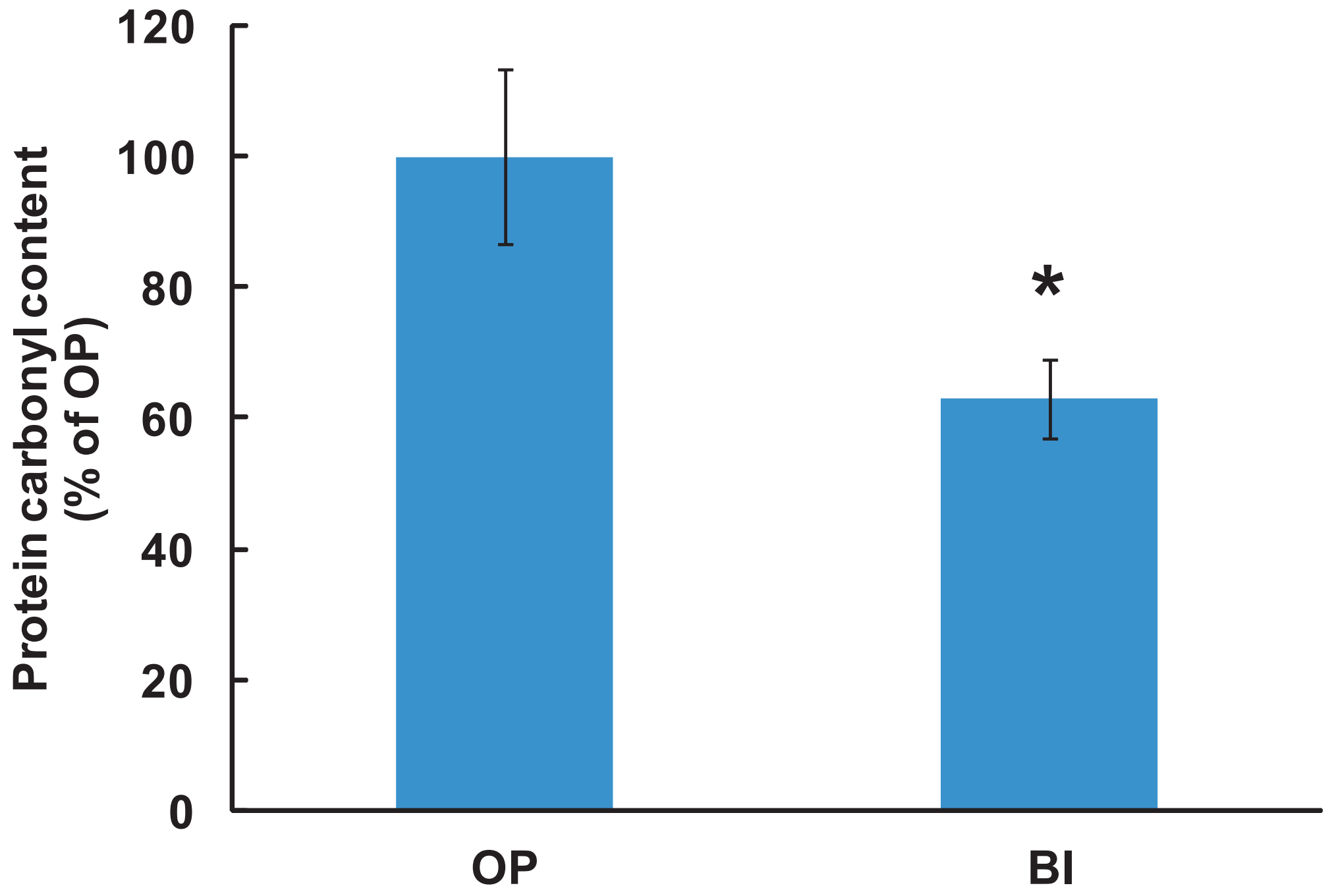




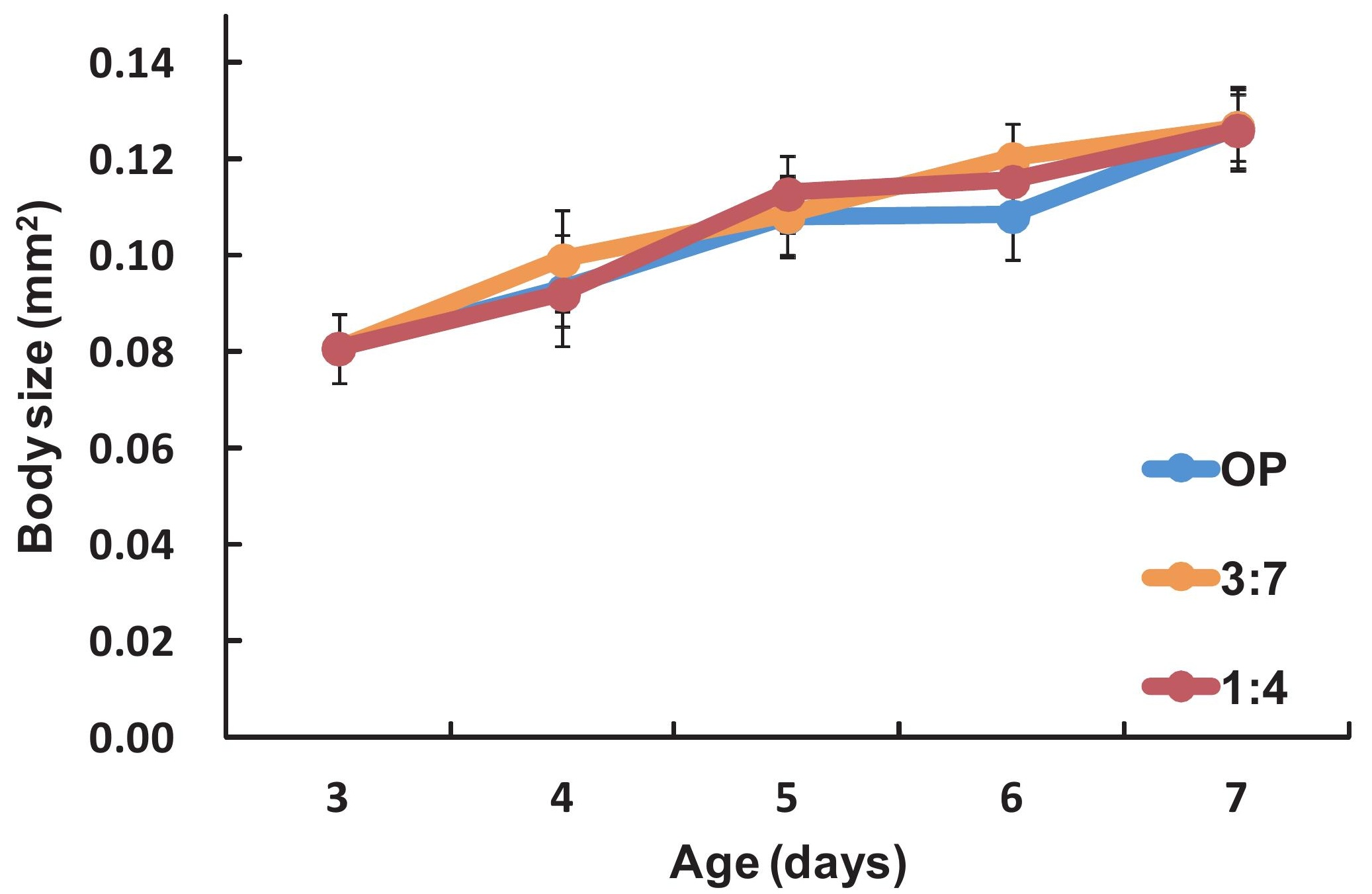




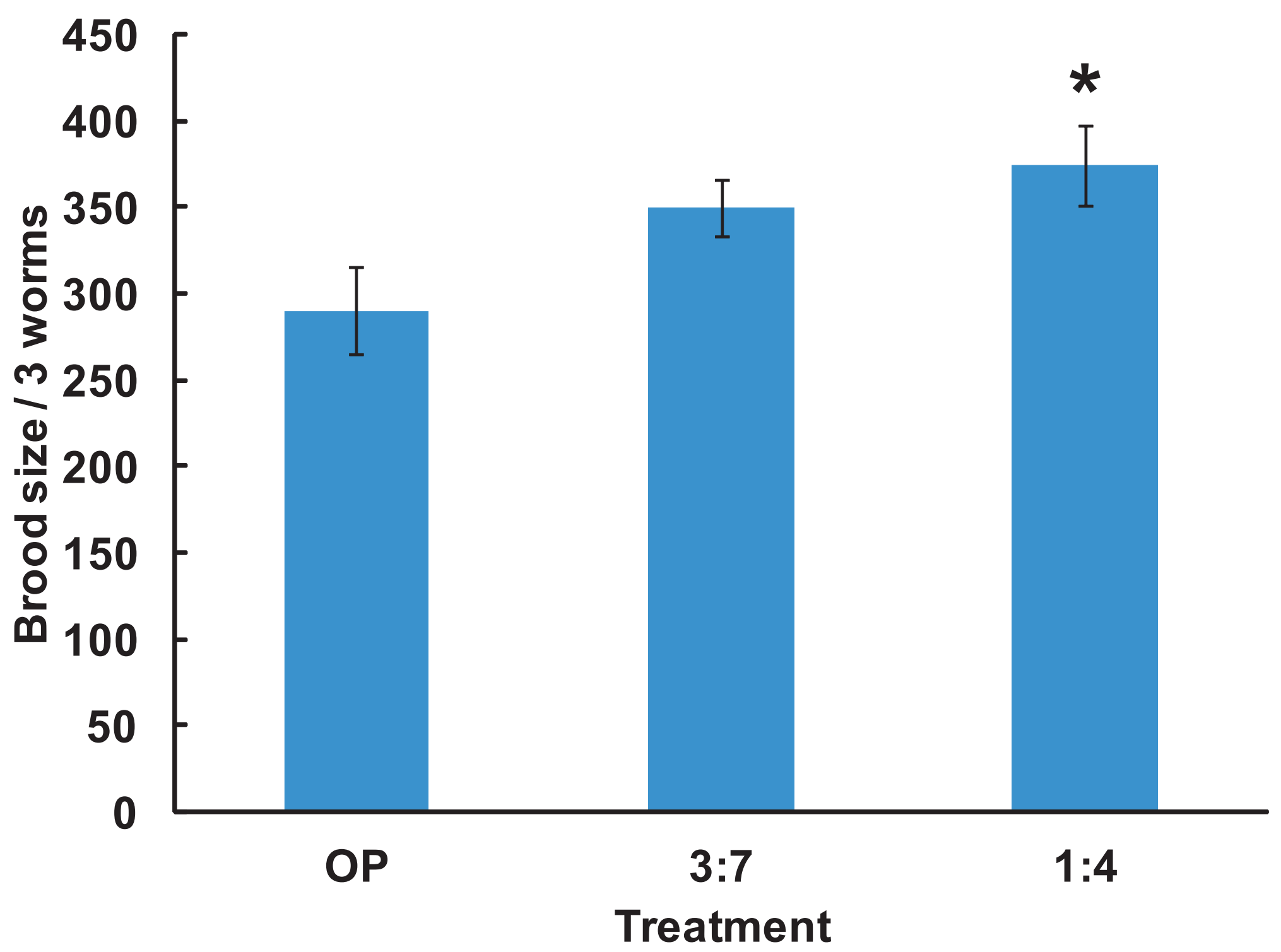




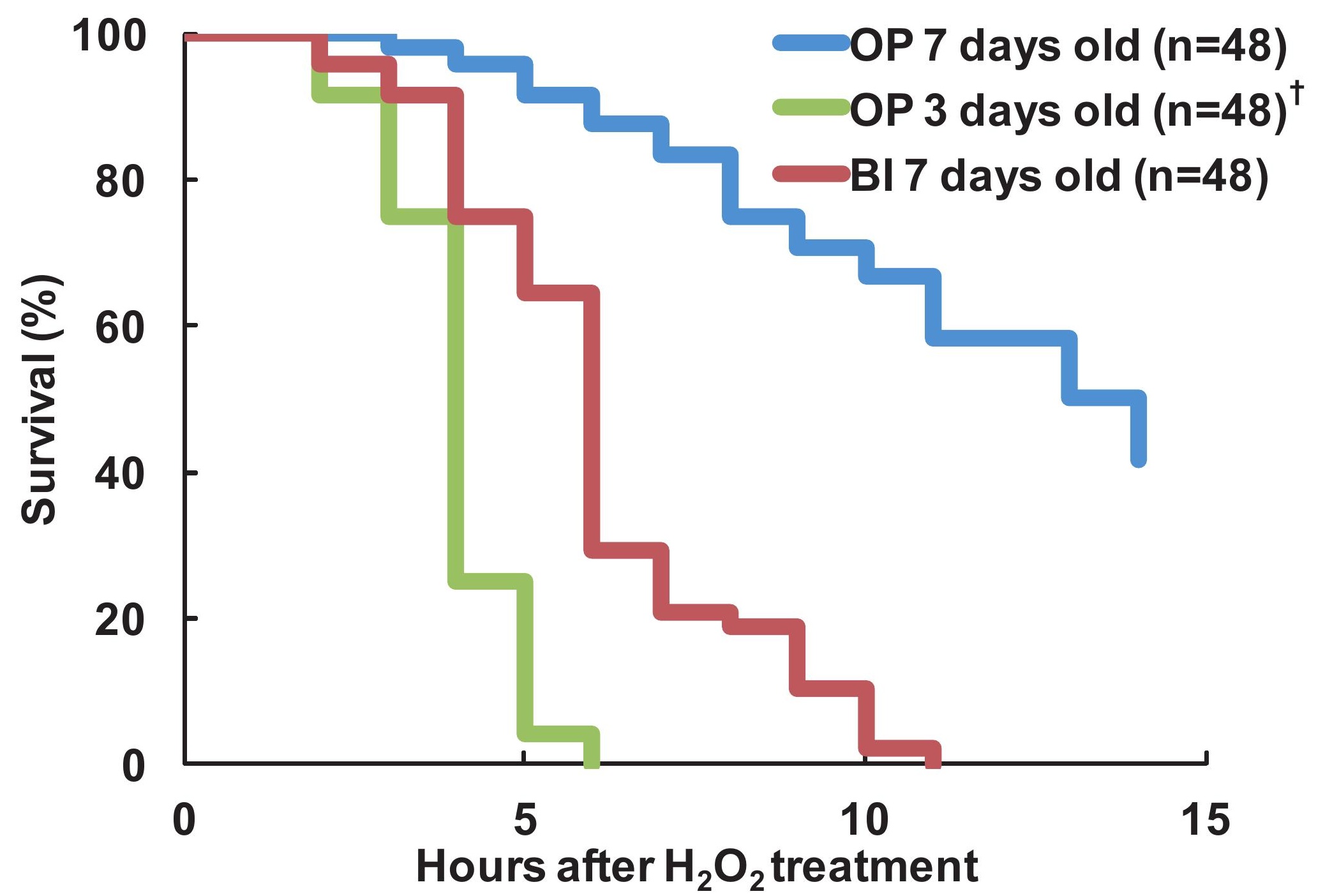




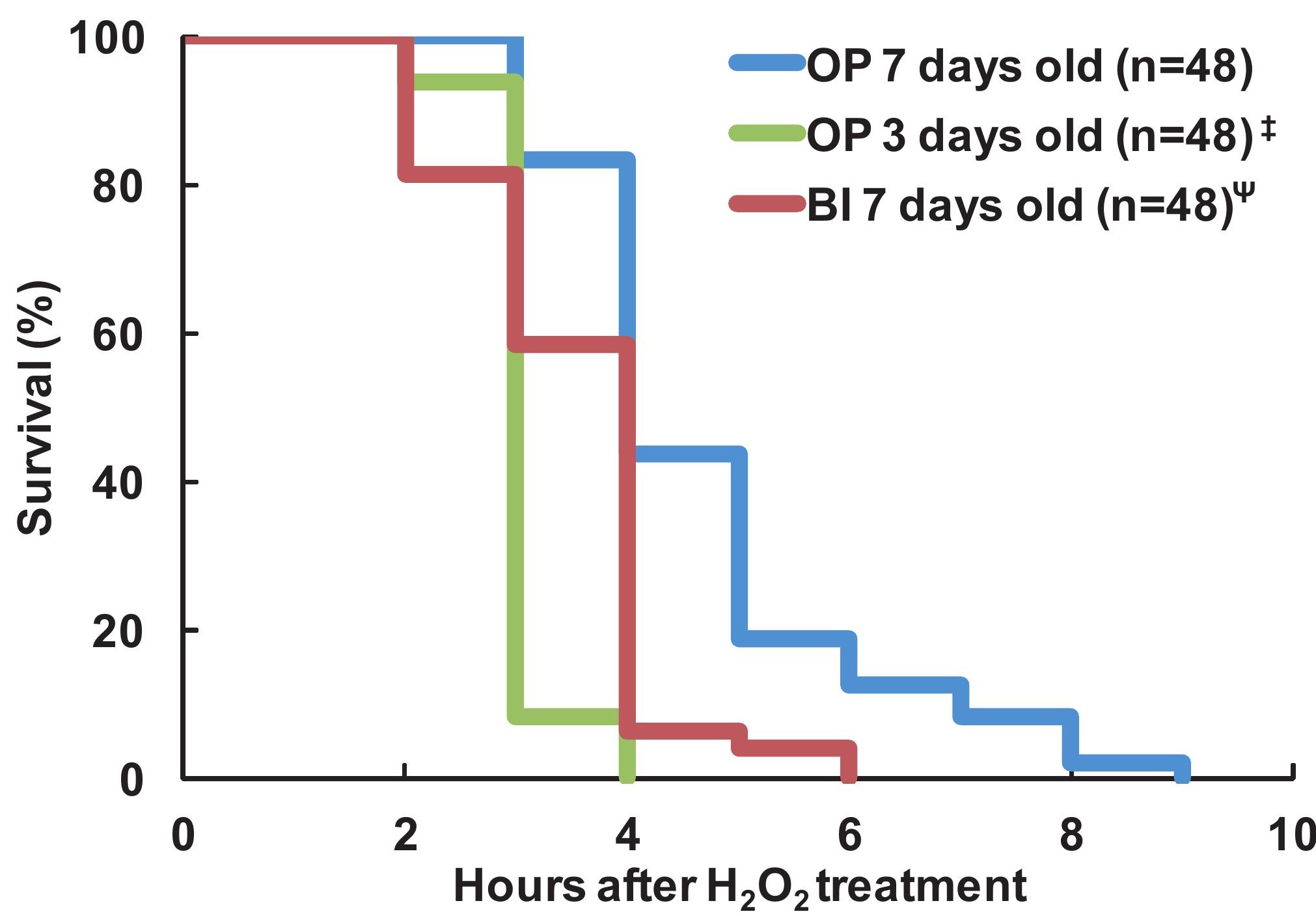




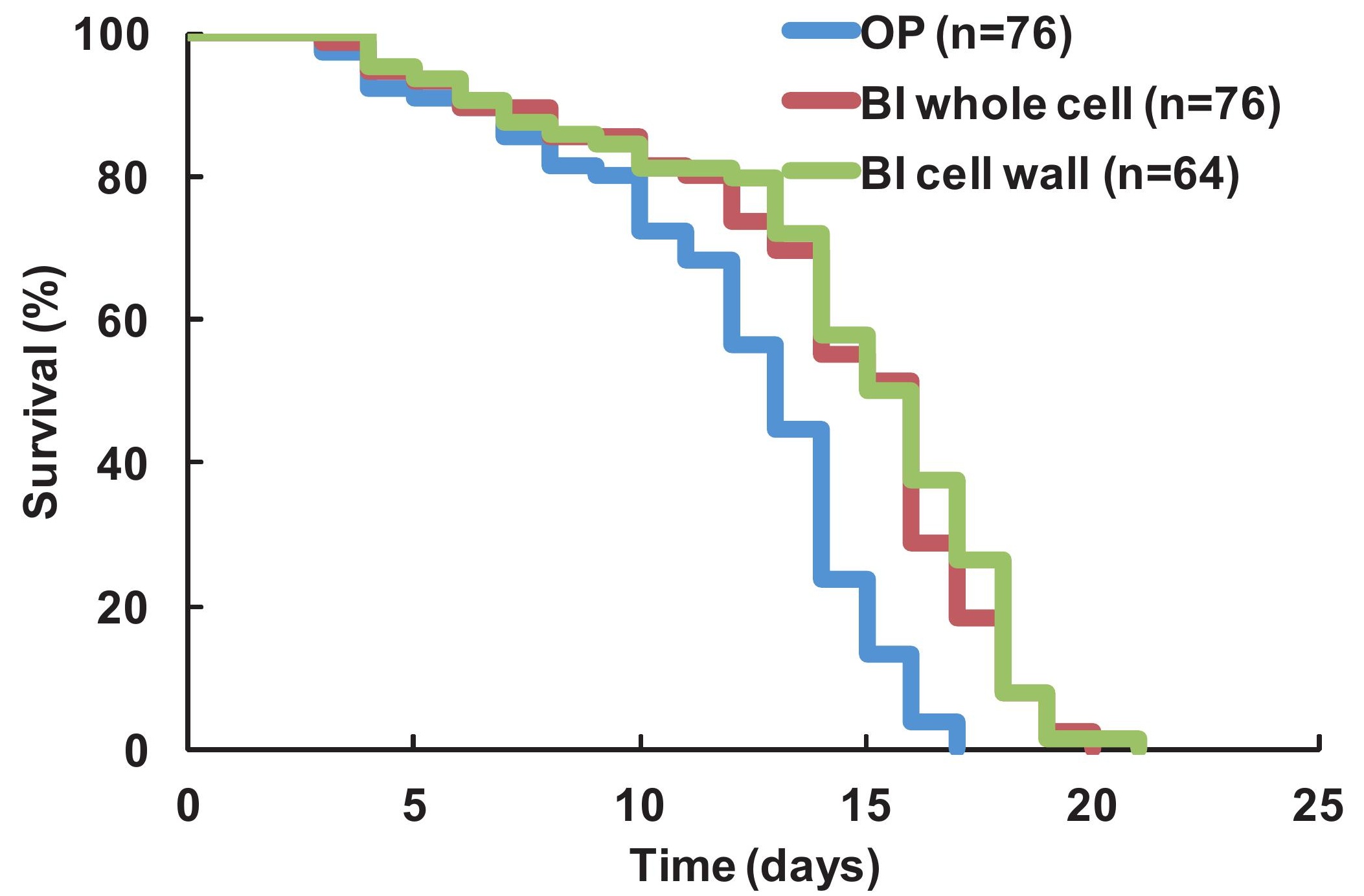




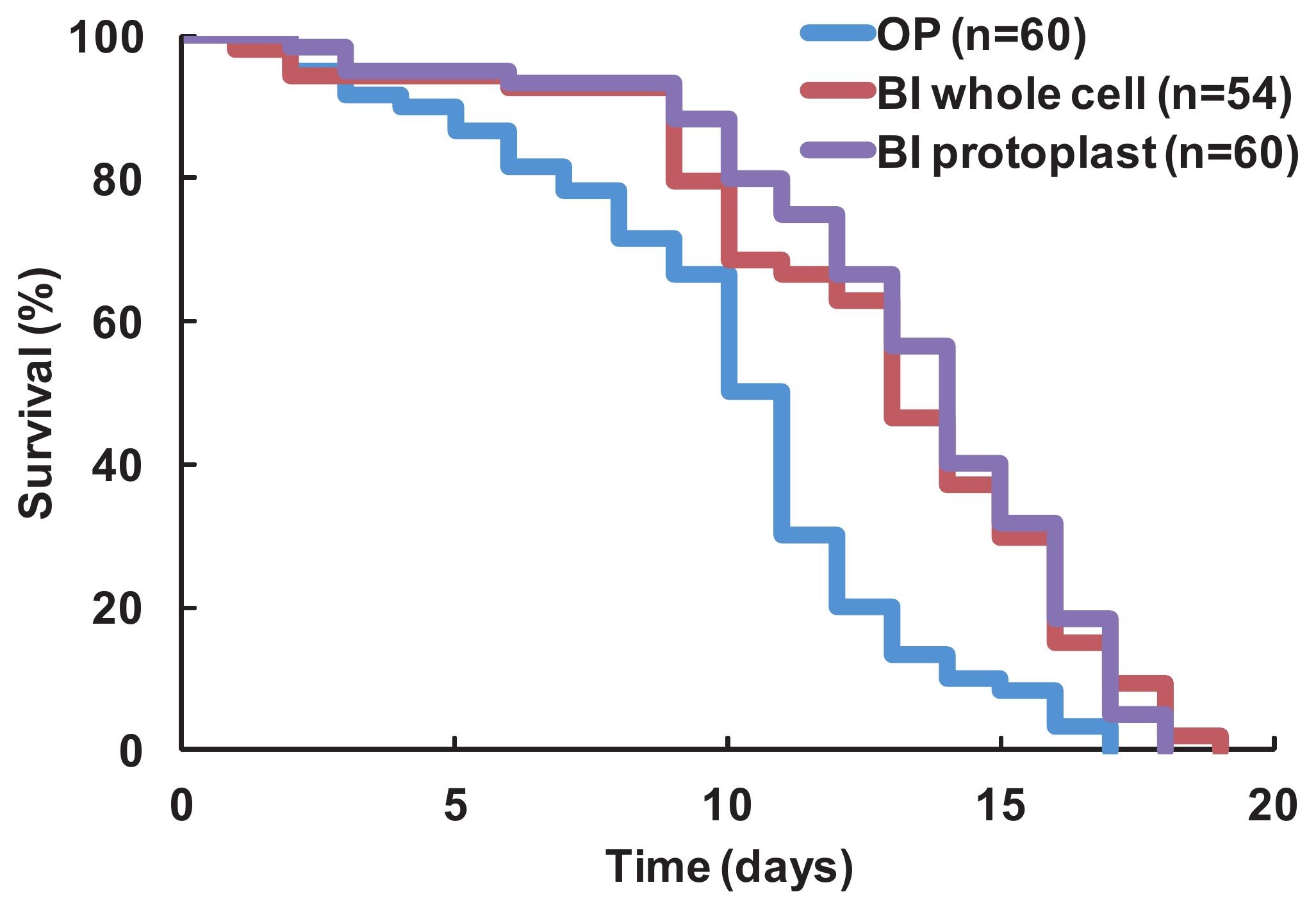




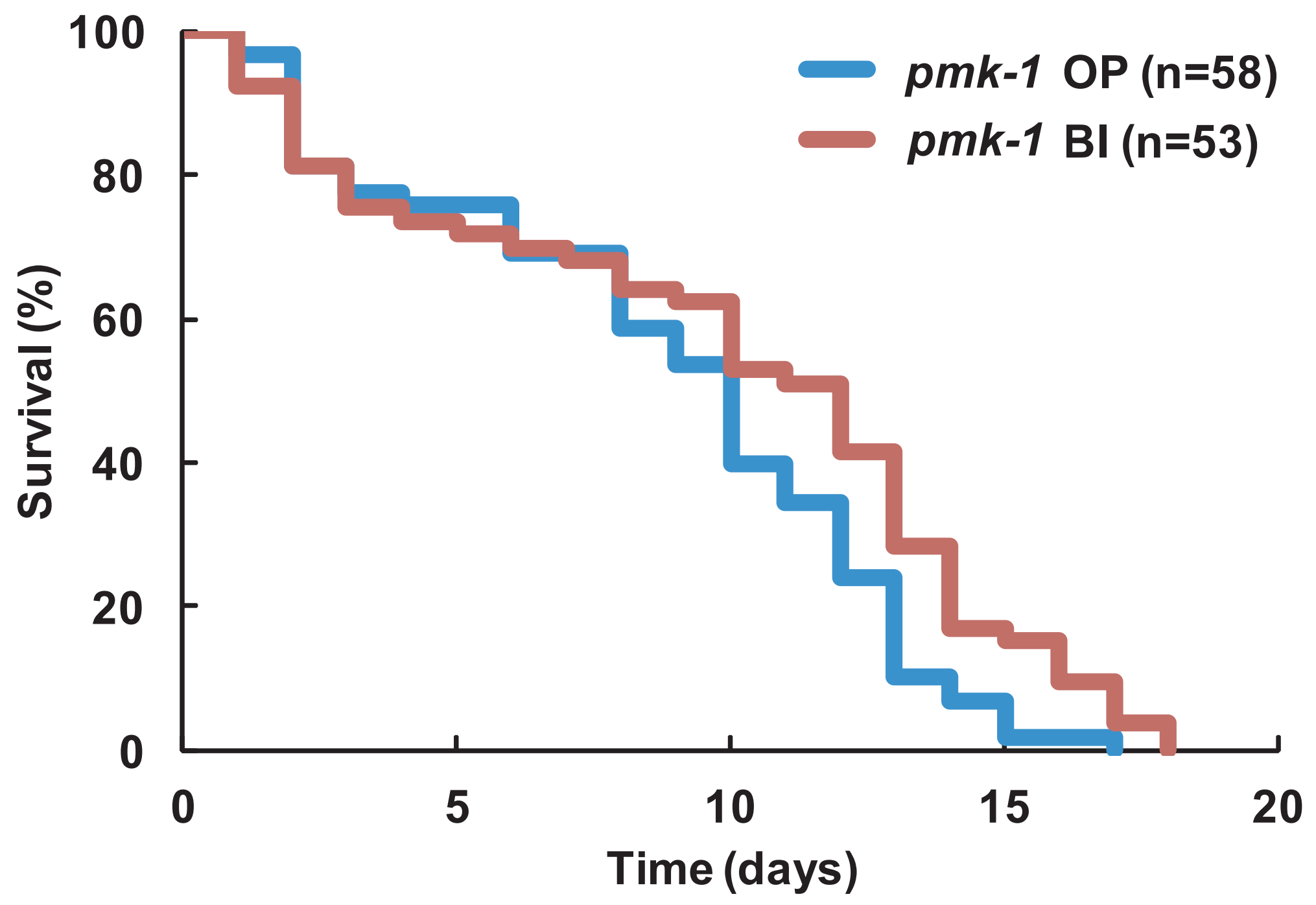




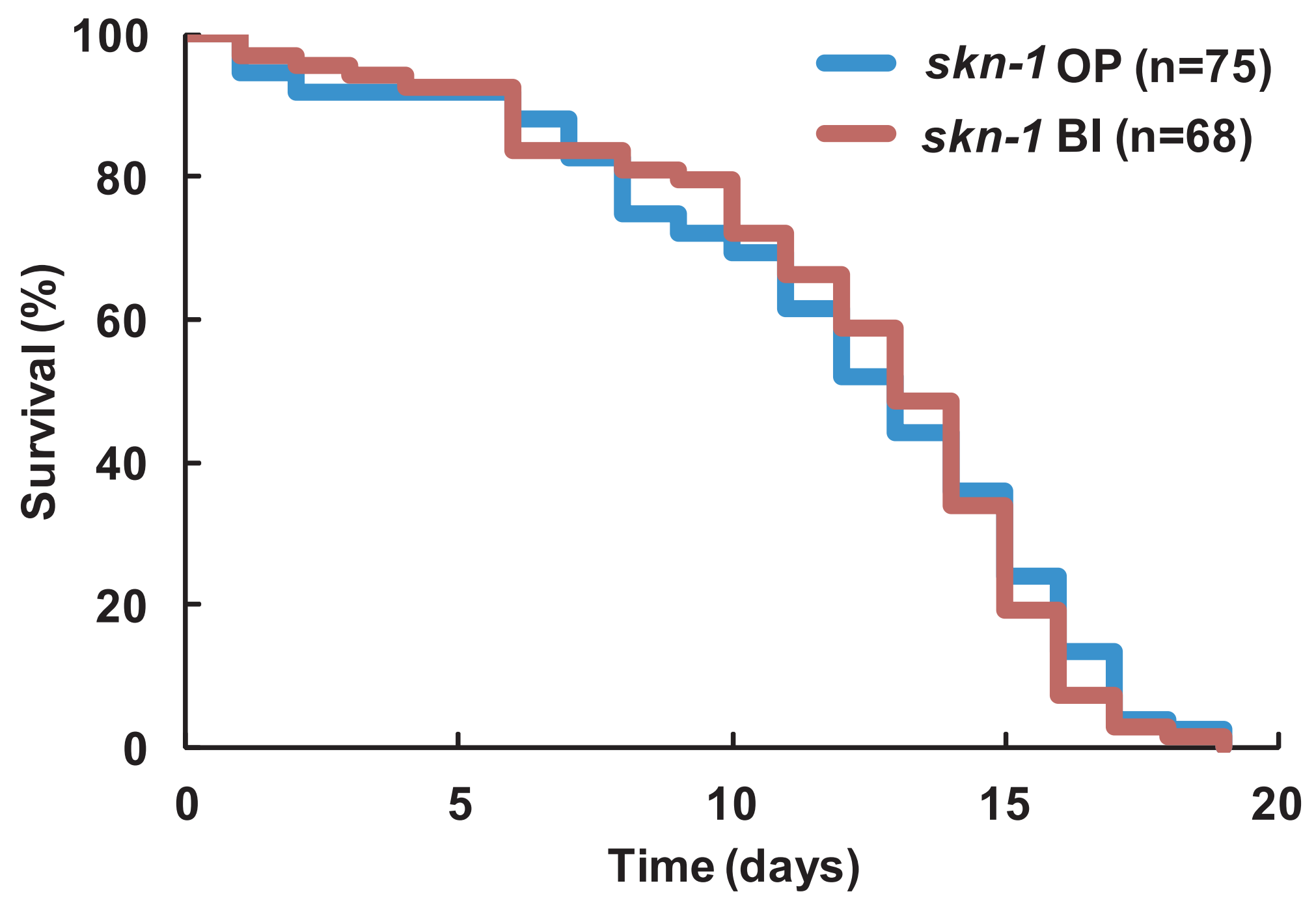




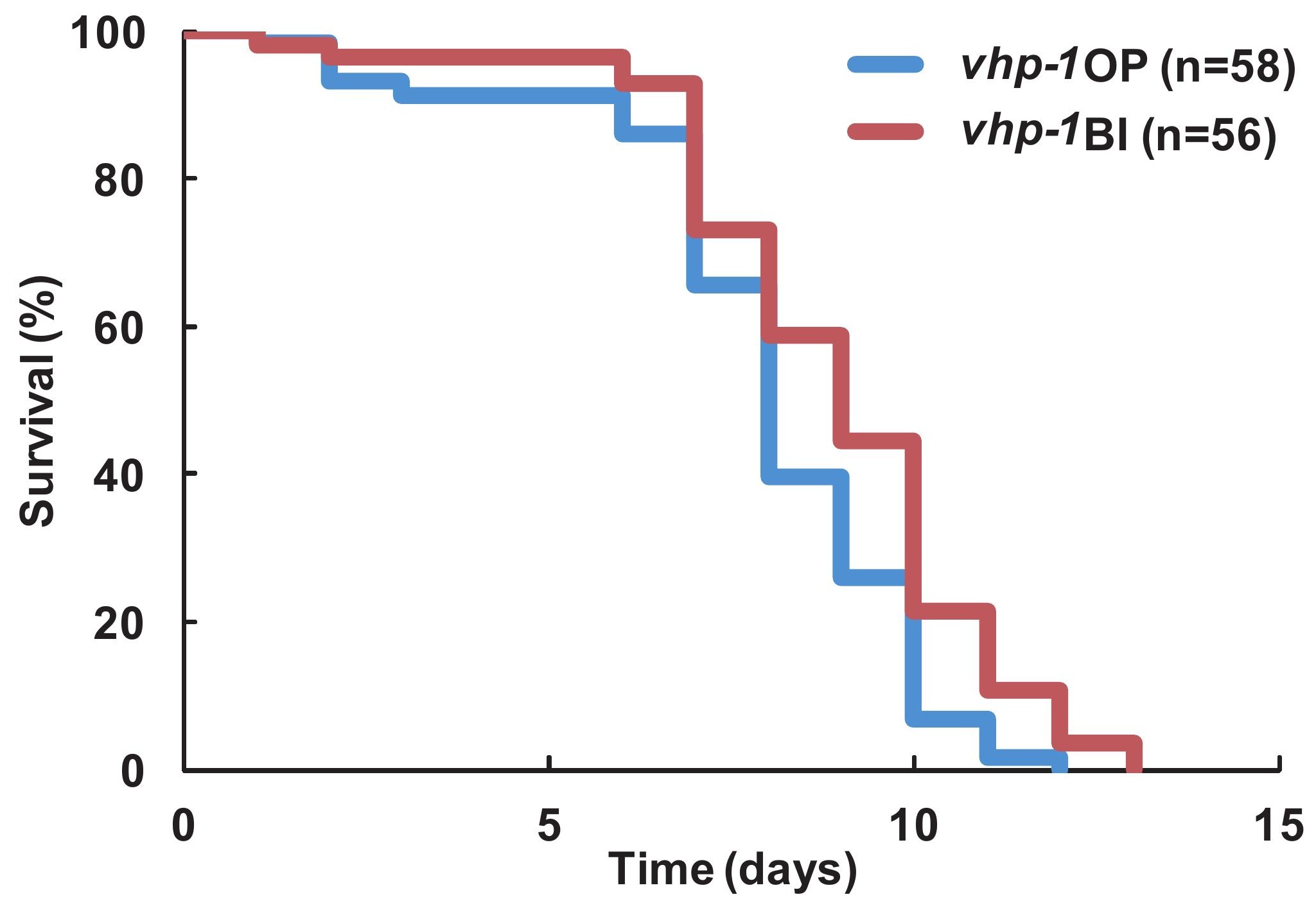




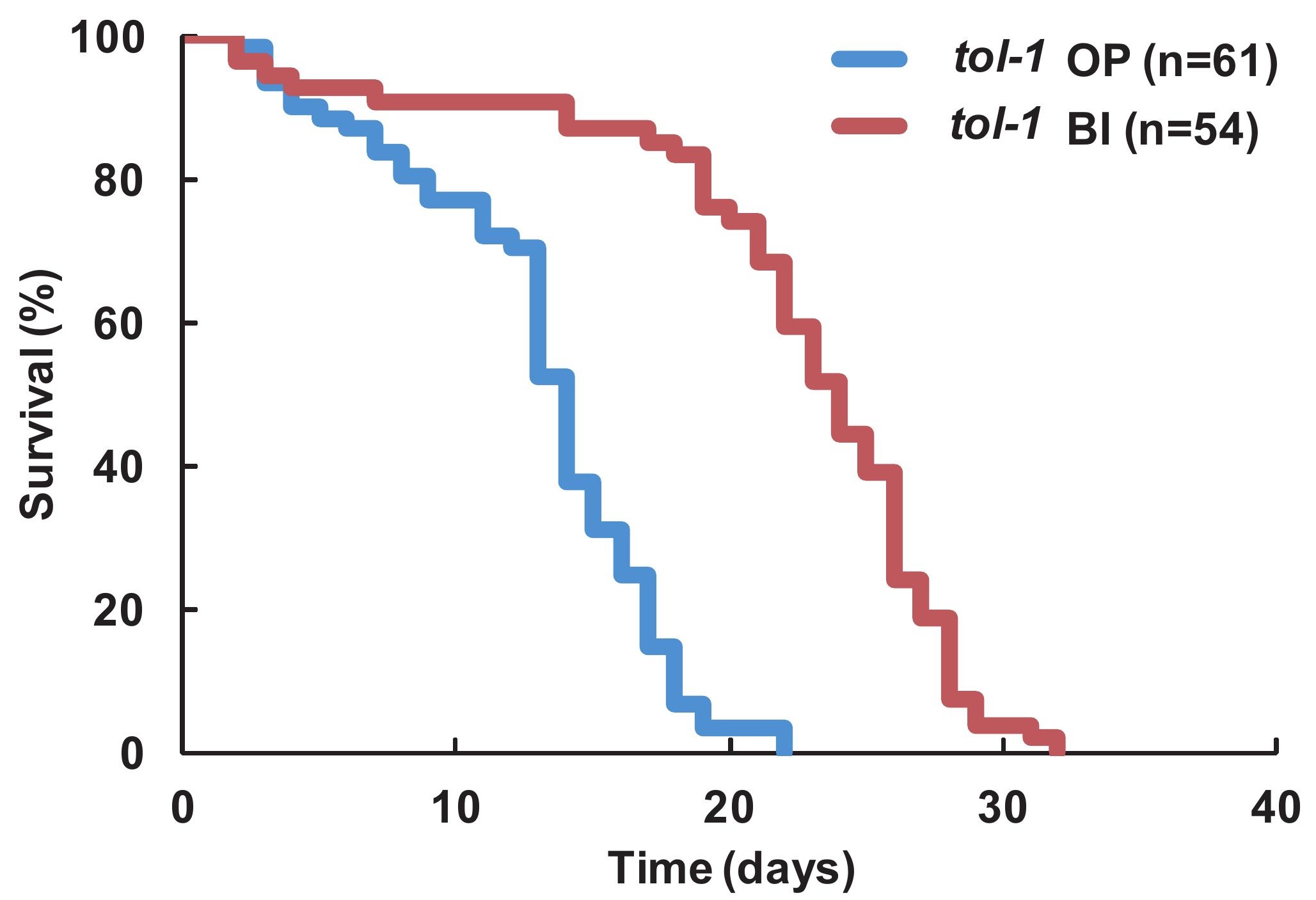




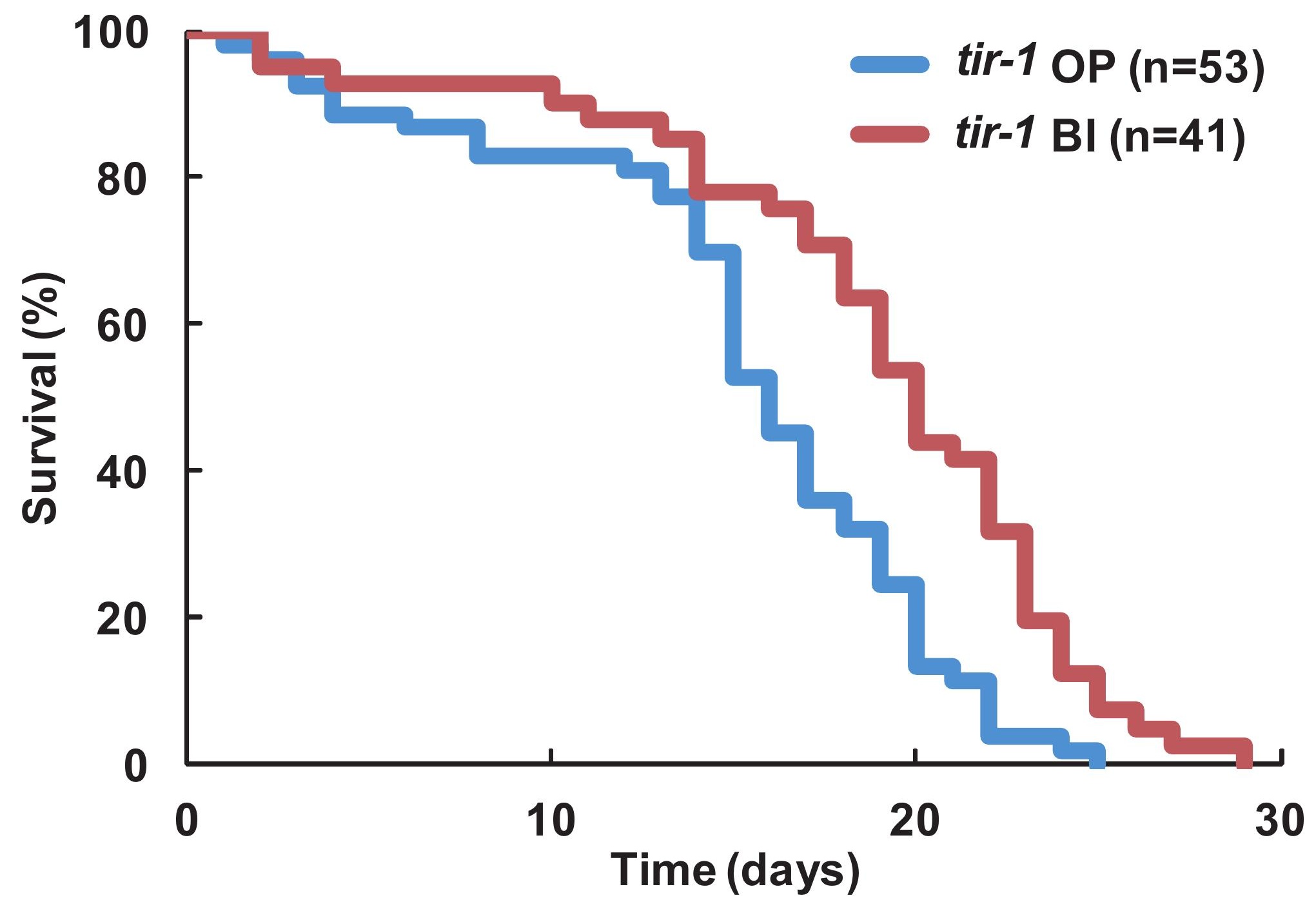




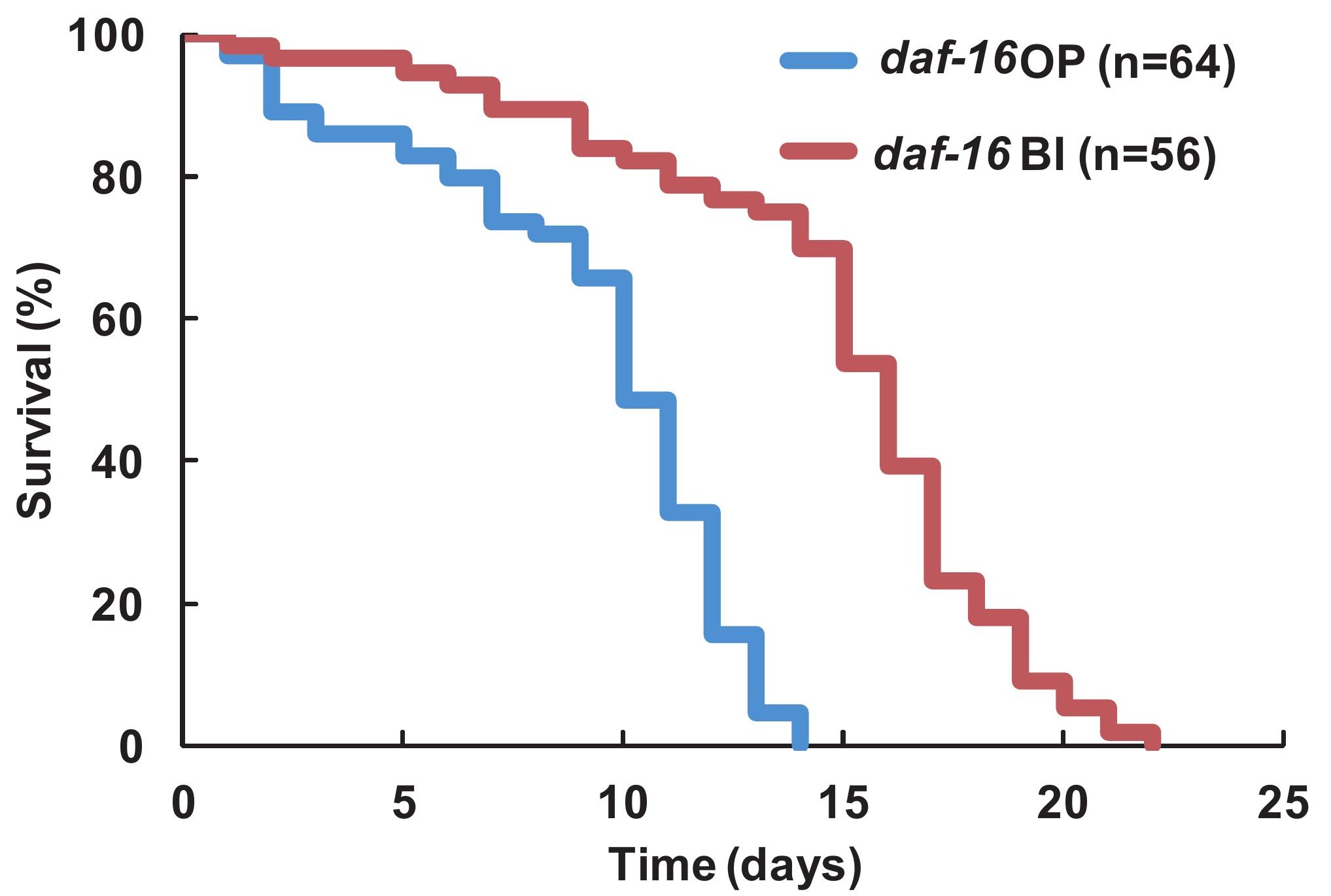




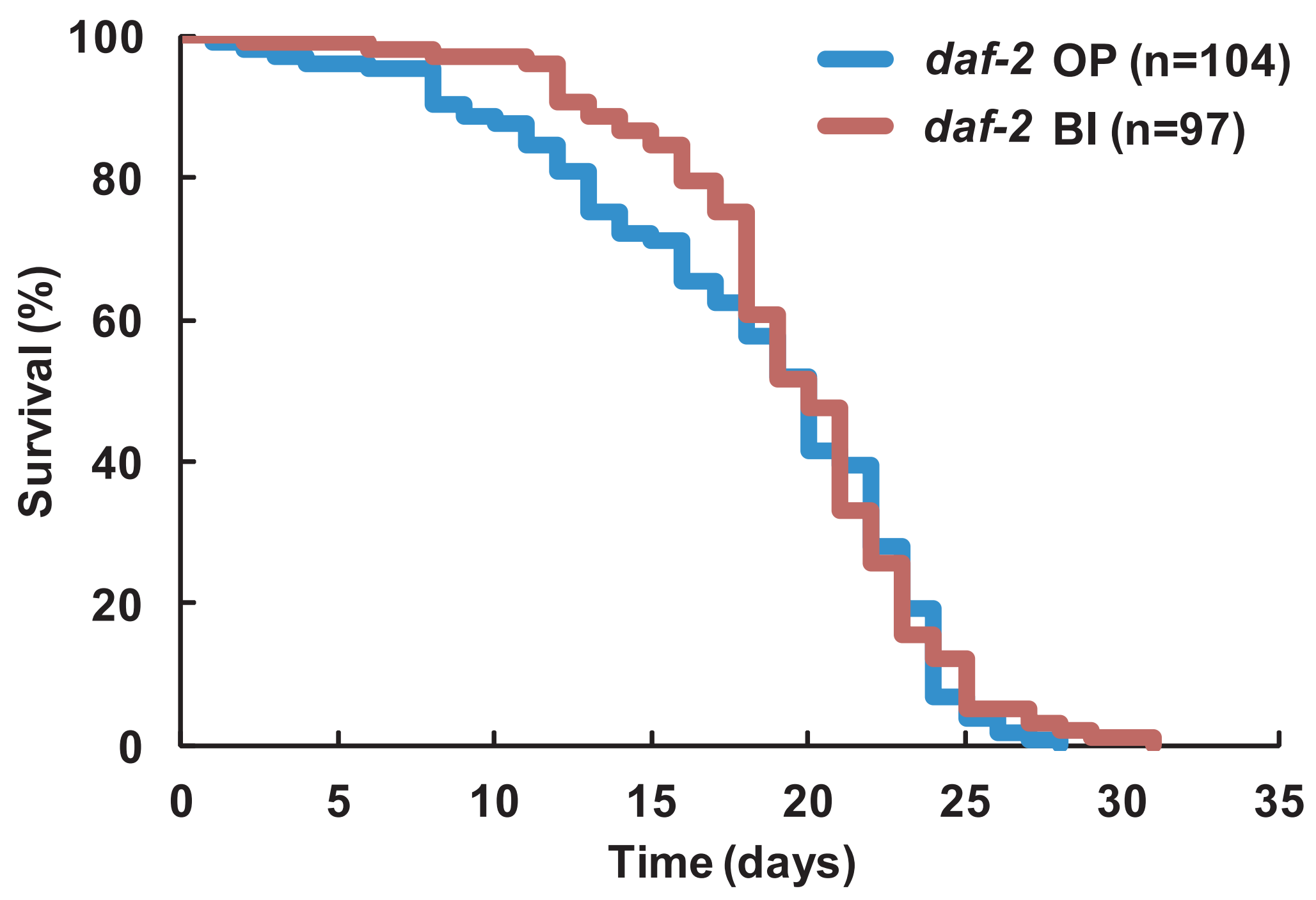




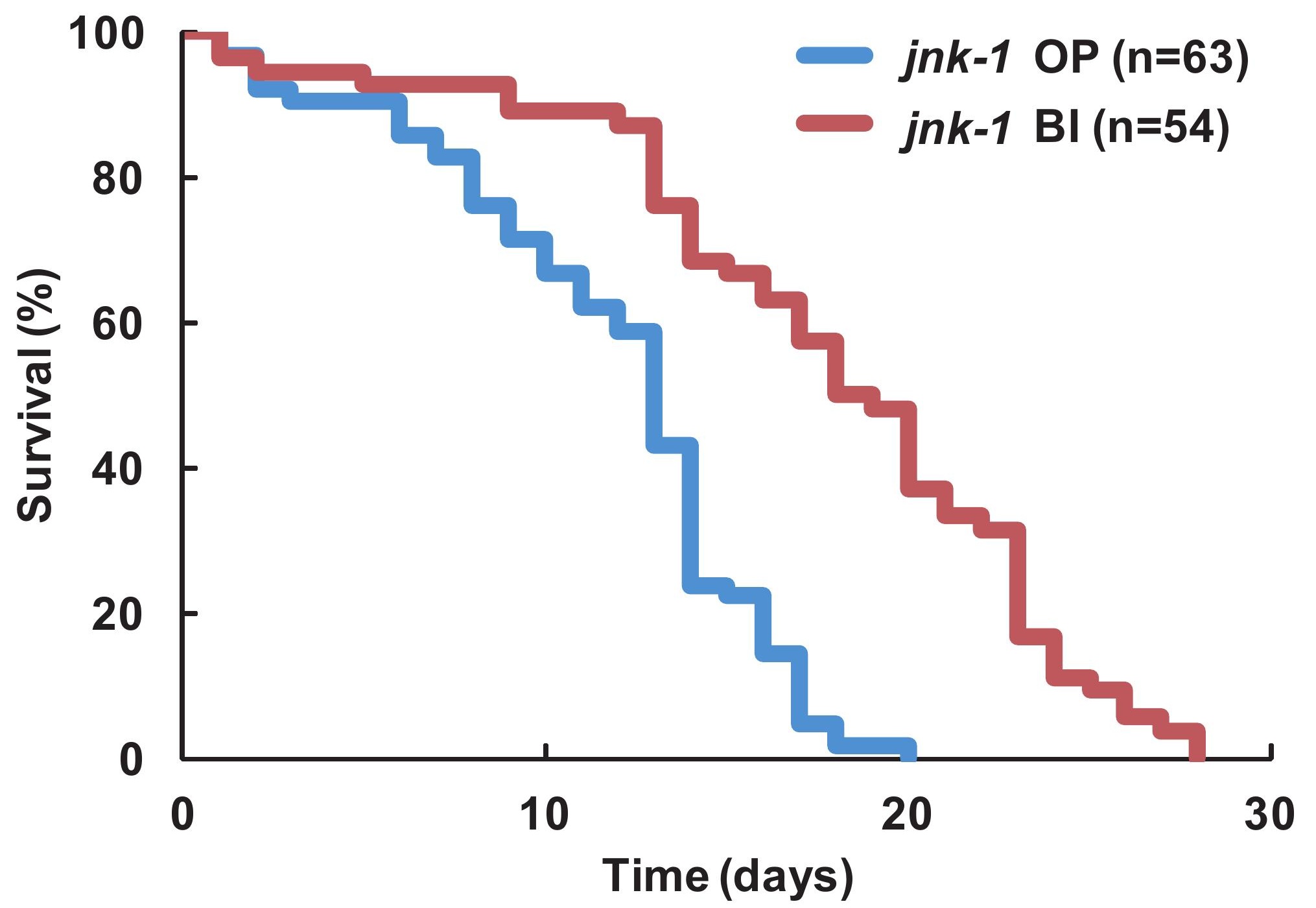




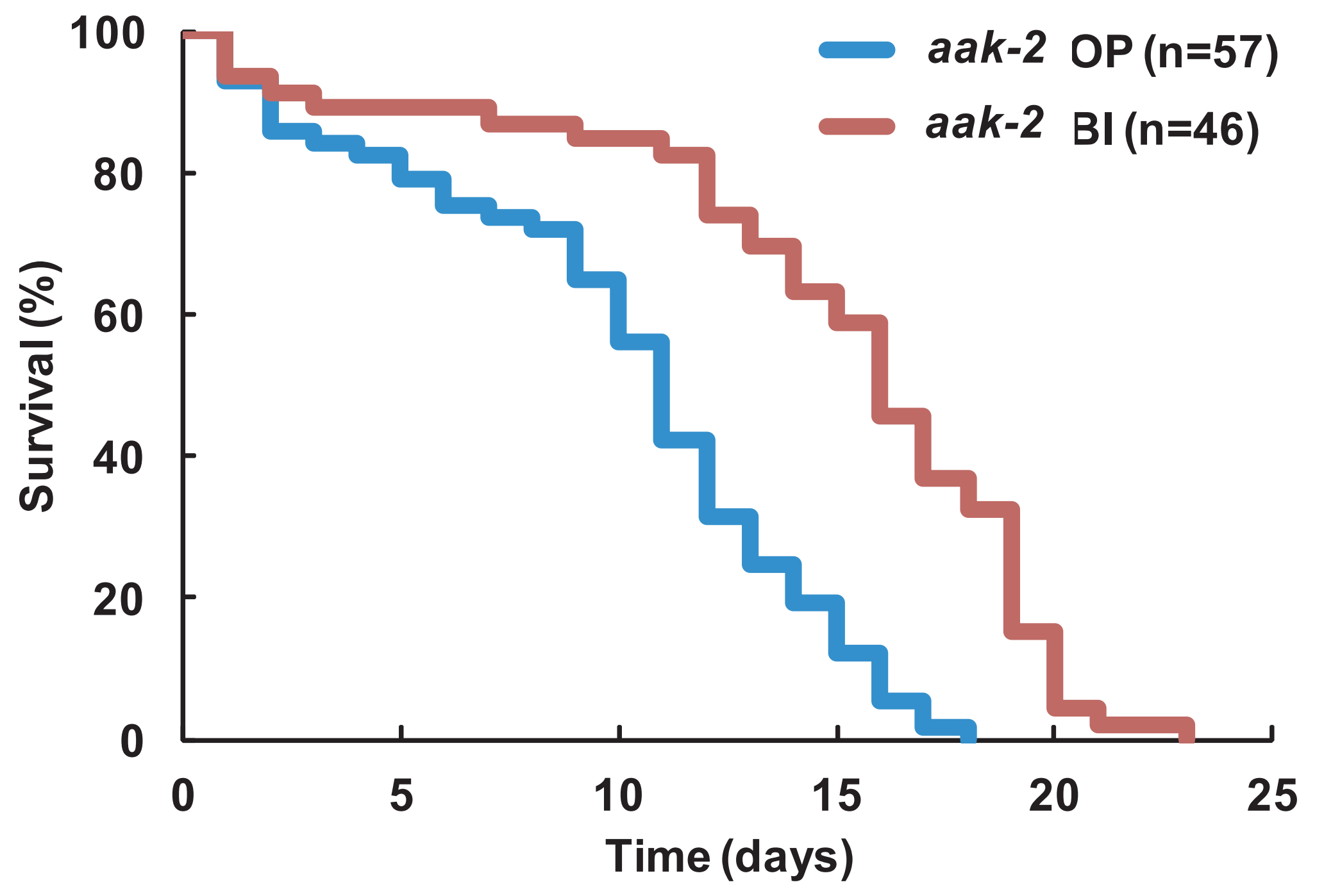




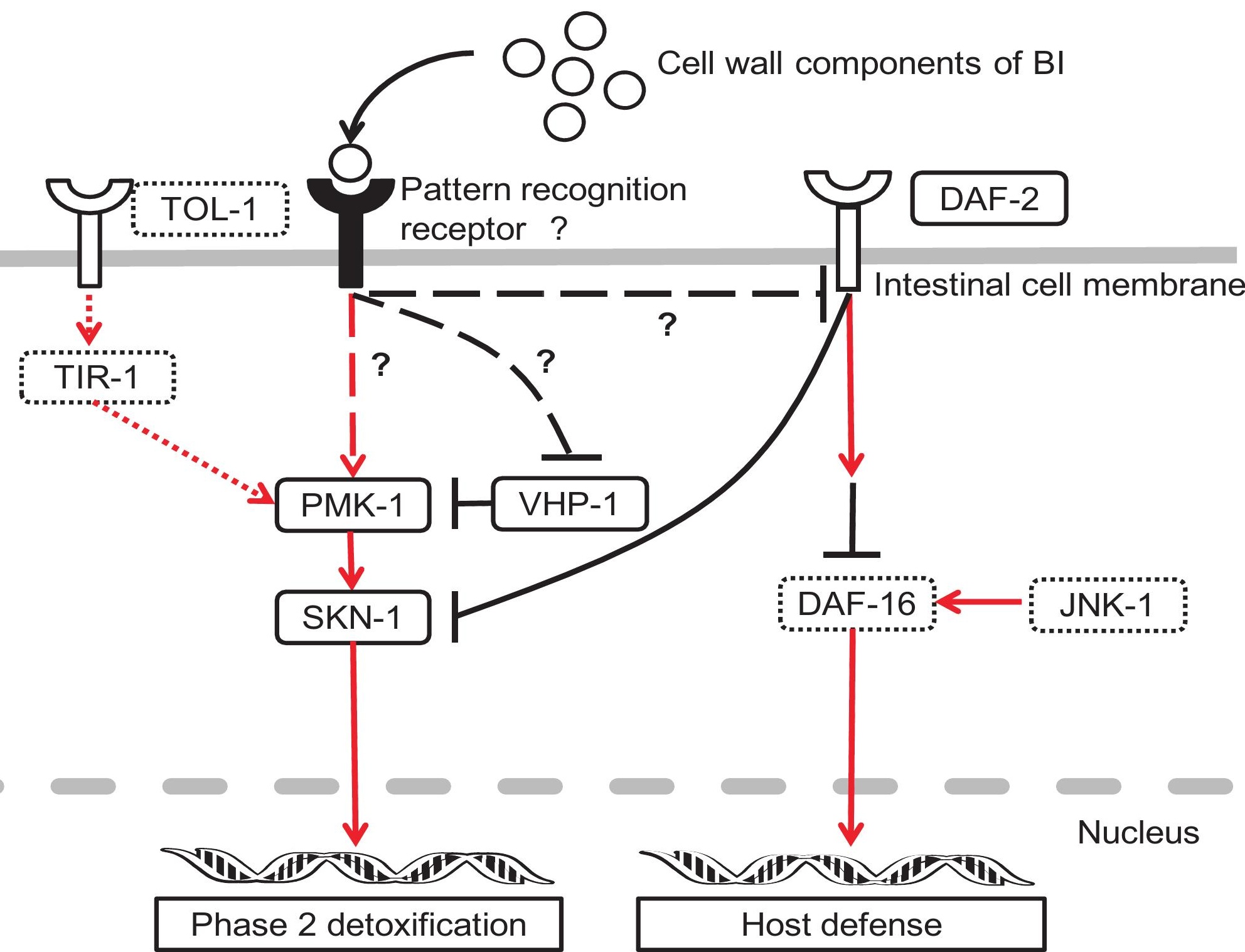

